Proceedings of the Edinburgh Mathematical Society (2002) 45, 467-490 (C)

DOI:10.1017/S0013091500000821 Printed in the United Kingdom

\title{
ON SOME CLASSES OF OPERATORS DETERMINED BY THE STRUCTURE OF THEIR MEMORY
}

\author{
MIKHAIL E. DRAKHLIN ${ }^{1}$, ARCADY PONOSOV ${ }^{2}$ AND EUGENE STEPANOV 3 \\ ${ }^{1}$ Research Institute, College of Judea and Samaria, \\ Kedumim-Ariel, DN Efraim 44837, Israel \\ ${ }^{2}$ Institutt for Matematiske Fag, Postboks 5035, \\ $N-1432$ Ås-NLH, Norway \\ ${ }^{3}$ Scuola Normale Superiore, Piazza dei Cavalieri 7, \\ 56126 Pisa, Italy (stepanov@cibs.sns.it)
}

(Received 20 July 2000)

\begin{abstract}
Two classes of nonlinear operators generalizing the notion of a local operator between ideal function spaces are introduced. The first class, called atomic, contains in particular all the linear shifts, while the second one, called coatomic, contains all the adjoints to former, and, in particular, the conditional expectations. Both classes include local (in particular, Nemytskiî) operators and are closed with respect to compositions of operators. Basic properties of operators of introduced classes in the Lebesgue spaces of vector-valued functions are studied. It is shown that both classes inherit from Nemytskir operators the properties of non-compactness in measure and weak degeneracy, while having different relationships of acting, continuity and boundedness, as well as different convergence properties. Representation results for the operators of both classes are provided. The definitions of the introduced classes as well as the proofs of their properties are based on a purely measure theoretic notion of memory of an operator, also introduced in this paper.
\end{abstract}

Keywords: local operators; Nemytskiǐ operators; shift operators; superposition operators; disjointness-preserving operators; ideal spaces

AMS 2000 Mathematics subject classification: Primary 47B38; 47A67; 34K05

\section{Introduction}

It is well known that many classical problems for differential equations, both ordinary and partial, usually involve only local operators between suitable function spaces. Two of the most famous examples of local operators, which justify the importance of this notion for applications, are linear differentiation operators between spaces of smooth functions or Sobolev spaces [19], and the nonlinear Nemytskiǐ (also called superposition) operator [1] usually defined between the spaces of summable functions (Lebesgue or Orlicz spaces). Another less well known but not less important example of a local operator which cannot be reduced to the above-mentioned ones is given by the stochastic integral defined over some suitable space of summable functions [14]. It is worth emphasizing that many 
general properties of classical differential equations are provided by the locality of the operators involved.

It is, however, well known that there are many applications involving operators which are intuitively 'almost local' and in fact have many very similar properties. Such operators are, for instance, linear weighted shifts, appearing in hereditary systems and functionaldifferential equations with deviating argument $[\mathbf{3}]$ as well as in many other applications. Since the general definition of a local operator [15] was formulated, several attempts have been made to obtain its reasonable generalization, which would cover many such applications, while not losing most of the important properties of local operators. It seems, however, that only the notion of disjointness-preserving $\left(D_{-}\right)$operators was rather successful in this sense. Moreover, even with the latter notion, most significant results were obtained only for the linear operator case $[\mathbf{2 0}]$, while we are inclined to think (and try to justify in this paper) that no reasonably 'nice' properties can be proved for general $D$-operators in the nonlinear case.

In this paper we point out two new extensions of the notion of a local operator in ideal function spaces. The first class of operators, called atomic, contains all the (linear) weighted shifts. The second one, in a certain sense dual to the first one, called coatomic, includes, in particular, conditional expectations and the adjoints of weighted shifts. Both classes include local operators and are closed with respect to composition of operators. We show that such operators arise naturally in the study of periodic solutions to stochastic differential equations, as well as in functional-differential equations. In this paper we study basic properties of the operators of the identified classes like acting, boundedness, continuity, compactness and convergence, and show that in many cases they just inherit the respective properties of local (and in particular, Nemytskiĩ) operators. However, we show by a number of counterexamples that the introduced objects are essentially new and in fact one may not just blindly extend the properties of local operators to such moregeneral classes. The terminology introduced is justified by the representation theorems we prove for both classes: for instance, it will be clear that atomic operators can be regarded as nonlinear integral operators generated by a random atomic measure.

We find it suitable to define the outlined two new classes of operators on the basis of the notion of the memory of an operator also introduced in this paper. Roughly speaking, a memory is a piece of information about the preimages the operator is able to 'remember' given a piece of information about images. This notion most naturally arises just from the definition of a local operator [15]. What is more, it will be easy to conclude that most of the common properties of local operators and the new introduced classes comes from nothing else but a measure-theoretic structure of their memory.

\section{Memory and comemory of an operator}

\subsection{Notation and preliminaries}

Let $(\Omega, \Sigma, \mu)$ be a measure space with a finite measure $\mu, \Sigma$ being a $\sigma$-algebra of its $\mu$-measurable subsets. A $\sigma$-ideal of subsets of measure zero (called below nullsets or $\mu$ nullsets, if the measure needs to be specified) will be denoted by $\Sigma^{0}$. Identifying in $\Sigma$ 
the sets which differ (in the sense of symmetric difference) by a nullset, we come to the $\sigma$-algebra $\tilde{\Sigma}:=\Sigma / \Sigma^{0}$ of classes of measurable sets modulo nullsets. It is customary to call $\tilde{\Sigma}$ a measure algebra of $\mu$ (see, for example, $\S 42$ of [16]). In general we identify the elements of $\tilde{\Sigma}$ with their representatives from $\Sigma$, but whenever there might be a possibility of confusion, we denote for $e \in \Sigma$ the respective equivalence class in $\tilde{\Sigma}$ by either $\tilde{e}$ or $[e]$. We also find it convenient to write $\left.x\right|_{e}=\left.y\right|_{e}$, if $x(\omega)=y(\omega)$ a.e. on the set $e \subset \Omega$.

By $L^{p}(\Omega, \Sigma, \mu ; \mathcal{X})$ we denote the classical Lebesgue-Bochner space of (classes of $\mu$ equivalent) functions summable with exponent $p>0$ over $\Omega$ with respect to the measure $\mu$ and taking values in some separable normed space $\mathcal{X}$. By $L^{0}(\Omega, \Sigma, \mu ; \mathcal{X})$, where $\mathcal{X}$ is a separable metric space, we denote the space of (classes of) $\mathcal{X}$-valued measurable functions equipped with the usual metric inducing the topology of convergence in measure. Whenever there is no possibility of confusion, the references to $\mathcal{X}, \Omega, \Sigma$ and/or $\mu$ will be omitted.

In some cases we assume $(\Omega, \Sigma, \mu)$ to be a standard measure space, where $\Omega$ is a Polish space [4], $\mu$ is a Borel measure on $\Omega, \Sigma$ is either the $\sigma$-algebra of Borel subsets of the space $\Omega$ or its $\mu$-completion.

The characteristic function of a subset $e \subset \Omega$ will be denoted by $\mathbf{1}_{e}(\omega)$ below, omitting the reference to $e$ when $e=\Omega$.

If $\left(\Omega_{1}, \Sigma_{1}, \mu_{1}\right)$ and $\left(\Omega_{2}, \Sigma_{2}, \mu_{2}\right)$ are two measure spaces, a map $F: \Sigma_{1} \rightarrow \Sigma_{2}$ is called a $\sigma$-homomorphism, if $F\left(\Omega_{1}\right)=\Omega_{2}, F\left(\Omega_{1} \backslash e\right)=\Omega_{2} \backslash F(e)$ whenever $e \in \Sigma_{1}$ and

$$
F\left(\bigsqcup_{i=1}^{\infty} e_{i}\right)=\bigsqcup_{i=1}^{\infty} F\left(e_{i}\right),
$$

for any pairwise disjoint collection of $\mu_{1}$-measurable sets $\left\{e_{i}\right\}_{i=1}^{\infty}$, where $\sqcup$ stands for the disjoint union. The $\sigma$-homomorphisms considered below will silently be assumed to be nullset preserving, that is,

$$
\mu_{2}\left(F\left(e_{1}\right)\right)=0 \quad \text { when } \mu_{1}\left(e_{1}\right)=0 .
$$

Thus $F$ naturally induces a $\sigma$-homomorphism $\tilde{F}: \tilde{\Sigma}_{1} \rightarrow \tilde{\Sigma}_{2}$ by the relationship $\tilde{F}\left(\tilde{e}_{1}\right):=$ $\left[F\left(e_{1}\right)\right]$.

\subsection{Memory and comemory}

Let $X_{i}:=L^{p_{i}}\left(\Omega_{i}, \Sigma_{i}, \mu_{i} ; \mathcal{X}_{i}\right), i=1,2$, and $0 \leqslant p_{i} \leqslant+\infty$. Consider an operator $T: X_{1} \rightarrow X_{2}$. We now introduce the concept of memory and the related concept of comemory.

Definition 2.1. We call the memory of an operator $T: X_{1} \rightarrow X_{2}$ on a set $e_{2} \in \Sigma_{2}$ the family of all possible $\tilde{e}_{1} \in \tilde{\Sigma}_{1}$ such that for any $x, y \in X_{1}$ satisfying $\left.x\right|_{e_{1}}=\left.y\right|_{e_{1}}$ it follows that $\left.T(x)\right|_{e_{2}}=\left.T(y)\right|_{e_{2}}$. In other words,

$$
\underset{T}{\operatorname{Mem}}\left(\tilde{e}_{2}\right):=\left\{\tilde{e}_{1} \in \tilde{\Sigma}_{1}:\left.x\right|_{e_{1}}=\left.y\right|_{e_{1}} \text { implies }\left.T(x)\right|_{e_{2}}=\left.T(y)\right|_{e_{2}}\right\},
$$


Similarly, the comemory of the operator $T$ on a set $e_{1} \in \Sigma_{1}$ is the family

$$
\operatorname{Comem}\left(\tilde{e}_{1}\right):=\left\{\tilde{e}_{2} \in \tilde{\Sigma}_{2}:\left.x\right|_{e_{1}}=\left.y\right|_{e_{1}} \text { implies }\left.T(x)\right|_{e_{2}}=\left.T(y)\right|_{e_{2}}\right\} .
$$

It is clear that $\tilde{e}_{1} \in \operatorname{Mem}_{T}\left(\tilde{e}_{2}\right)$, if and only if $\tilde{e}_{2} \in \operatorname{Comem}_{T}\left(\tilde{e}_{1}\right)$.

Example 2.2. Define a shift operator $T_{g}: L^{0}\left(\Omega_{1} ; \mathcal{X}\right) \rightarrow L^{0}\left(\Omega_{2} ; \mathcal{X}\right)$ (sometimes also called inner superposition) by the formula

$$
\left(T_{g} x\right)\left(\omega_{2}\right):=x\left(g\left(\omega_{2}\right)\right),
$$

where $g: \Omega_{2} \rightarrow \Omega_{1}$ is a given $\left(\Sigma_{2}, \Sigma_{1}\right)$-measurable function. For this operator to be well defined on the classes of measurable functions we require

$$
\mu_{2}\left(g^{-1}\left(e_{1}\right)\right)=0 \quad \text { for } e_{1} \in \Sigma_{1}, \quad \mu_{1}\left(e_{1}\right)=0 .
$$

Then

$$
\underset{T_{g}}{\operatorname{Mem}}\left(\tilde{e}_{2}\right)=\left\{\tilde{e}_{1} \in \tilde{\Sigma}_{1}: e_{1} \supset g\left(e_{2}\right)\right\} .
$$

Example 2.3. Let $\Omega \subset \mathbb{R}^{n}$ be a compact set supplied with the $n$-dimensional Lebesgue measure $\mu, \Sigma$ being the Lebesgue $\sigma$-algebra. We define an operator $T: L^{1}(\Omega) \rightarrow L^{1}(\Omega)$ by the formula

$$
(T x)(\omega):=\int_{\Omega} x(s) \mathrm{d} s \cdot \mathbf{1}(\omega),
$$

i.e. $T$ maps every function into its integral. Then

$$
\underset{T}{\operatorname{Mem}}(\tilde{\mathcal{E}})= \begin{cases}\{\tilde{\Omega}\}, & \mu(\mathcal{E}) \neq 0, \\ \tilde{\Sigma}, & \mu(\mathcal{E})=0 .\end{cases}
$$

\subsection{Local and Nemytskiǐ operators}

Let $X_{i}=L^{0}\left(\Omega, \Sigma, \mu ; \mathcal{X}_{i}\right), i=1,2$.

Definition 2.4. An operator $T: X_{1} \rightarrow X_{2}$ is called local, if

$$
\tilde{e} \in \underset{T}{\operatorname{Mem}}(\tilde{e})
$$

for all $e \in \Sigma$, that is, if $\left.x\right|_{e}=\left.y\right|_{e}$ implies $\left.T(x)\right|_{e}=\left.T(y)\right|_{e}$ for all $x, y \in X_{1}$.

The above general definition reformulates the classical one due to Shragin [15] using the notion of memory. The following example is also classical and in fact motivated the study of local operators.

Example 2.5. Let $\mathcal{X}_{1}$ and $\mathcal{X}_{2}$ be separable metric spaces, $f: \Omega \times \mathcal{X}_{1} \rightarrow \mathcal{X}_{2}$ be a sup-measurable function (i.e. $f(\cdot, x(\cdot))$ is $\mu$-measurable whenever $x(\cdot)$ is $\mu$-measurable). Then the Nemytskiǐ operator $N_{f}: L^{0}\left(\Omega ; \mathcal{X}_{1}\right) \rightarrow L^{0}\left(\Omega ; \mathcal{X}_{2}\right)$ (commonly also known by the name superposition operator $[\mathbf{1}])$, defined by

$$
\left(N_{f} x\right)(\omega):=f(\omega, x(\omega)),
$$


is local. If $f: \Omega \times \mathcal{X}_{1} \rightarrow \mathcal{X}_{2}$ is a Carathéodory function (i.e. $f(\omega, \cdot)$ is continuous for $\mu$-almost every $\omega \in \Omega$ and $f(\cdot, x)$ is $\mu$-measurable for all $\left.x \in \mathcal{X}_{1}\right)$, then the Nemytskii operator $N_{f}$ becomes continuous in measure (i.e. as an operator in $L^{0}$ ).

Much research has now been done on Nemytskiǐ operators, especially in ideal function spaces. The reader is referred to the recent monographs $[\mathbf{1}]$ and $[\mathbf{1 7}]$ for the collection of results on this topic. It is known, however, that in general local operators over spaces of vector-valued summable functions are not reduced to Nemytskir operators, although the former inherit many properties of the latter.

\section{Atomic operators}

Now we introduce another definition generalizing the notion of a local operator. Here $X_{i}:=L^{p_{i}}\left(\Omega_{i}, \Sigma_{i}, \mu_{i}: \mathcal{X}_{i}\right), i=1,2$, and $0 \leqslant p_{i} \leqslant+\infty$.

Definition 3.1. An operator $T: X_{1} \rightarrow X_{2}$ is called atomic, if there is a $\sigma$-homomorphism $F: \Sigma_{1} \rightarrow \Sigma_{2}$, satisfying

$$
\left[F\left(e_{1}\right)\right] \in \underset{T}{\operatorname{Comem}}\left(\tilde{e}_{1}\right)
$$

for all $e_{1} \in \Sigma_{1}$, that is, if $\left.x\right|_{e_{1}}=\left.y\right|_{e_{1}}$ implies $\left.T(x)\right|_{F\left(e_{1}\right)}=\left.T(y)\right|_{F\left(e_{1}\right)}$ for all $x, y \in X_{1}$.

Obviously, every local operator is atomic. However, the class of atomic operators is richer, as one can conclude from the following example.

Example 3.2. Every shift operator (see Example 2.2) $T_{g}: L^{0}\left(\Omega_{1} ; \mathcal{X}\right) \rightarrow L^{0}\left(\Omega_{2} ; \mathcal{X}\right)$ is atomic. To show this, it is enough to set $F\left(e_{1}\right):=g^{-1}\left(e_{1}\right)$.

The class of atomic operators is obviously closed under compositions.

Example 3.3. Let $\Sigma_{2}$ be a $\sigma$-algebra of Borel subsets (or its $\mu_{2}$-completion) of a metric space $\Omega_{2}, \mathcal{X}_{1}$ and $\mathcal{X}_{2}$ be separable metric spaces, $f: \Omega_{2} \times \mathcal{X}_{1} \rightarrow \mathcal{X}_{2}$ be a Carathéodory function, and $g: \Omega_{2} \rightarrow \Omega_{1}$ be a $\left(\Sigma_{2}, \Sigma_{1}\right)$-measurable function satisfying (2.2). Then the operator $T: L^{0}\left(\Omega_{1} ; \mathcal{X}_{1}\right) \rightarrow L^{0}\left(\Omega_{2} ; \mathcal{X}_{2}\right)$ defined by the relationship

$$
(T x)\left(\omega_{2}\right):=f\left(\omega_{2}, x\left(g\left(\omega_{2}\right)\right)\right)
$$

is atomic as a composition of a Nemytskii operator $N_{f}$, and a shift $T_{g}$.

The example below shows, however, that unlike local operators the class of atomic operators is not closed under finite sums, even if the latter are well defined.

Example 3.4. Let $\Omega=(0,1), \Sigma$ be the $\sigma$-algebra of Lebesgue measurable subsets, $\mu$ being the one-dimensional Lebesgue measure. Consider the shift operator $T_{g}: L^{2}(\Omega) \rightarrow$ $L^{2}(\Omega)$ defined by the formula $\left(T_{g} x\right)(\omega):=x(1-\omega)$. We show that $T:=\operatorname{Id}+T_{g}$ is not atomic even though both summands are, where Id stands for the identity in $L^{2}$. Indeed, for an operator to be atomic, it is necessary that for any disjoint $e_{1}^{1}$ and $e_{1}^{2}$ in $\Sigma_{1}$ there exist such disjoint $e_{2}^{1}$ and $e_{2}^{2}$ in $\Sigma_{2}$ with $e_{1}^{1} \sqcup e_{1}^{2}=\Omega_{1}$ that $\tilde{e}_{2}^{1} \in \operatorname{Comem}_{T}\left(\tilde{e}_{1}^{1}\right), \tilde{e}_{2}^{2} \in \operatorname{Comem}_{T}\left(\tilde{e}_{1}^{2}\right)$ 
and $e_{2}^{1} \sqcup e_{2}^{2}=\Omega_{2}$. It is enough hence to take $e_{1}^{1}:=\left(0, \frac{1}{2}\right]$ and $e_{1}^{2}:=\left(\frac{1}{2}, 1\right)$ and observe that

$$
\underset{T}{\operatorname{Comem}}\left(\tilde{e}_{1}^{1}\right)=\underset{T}{\operatorname{Comem}}\left(\tilde{e}_{1}^{2}\right)=\tilde{\emptyset},
$$

so there is no possible way to choose sets $e_{2}^{1}$ and $e_{2}^{2}$ in $\Sigma_{2}$ with the aforementioned properties.

We now present the key example of this section, which shows how atomic operators arise naturally in a periodic problem for a stochastic differential equation.

Example 3.5. Let $(\Omega, \Sigma, \mathbb{P})$ be a probability space and $\Sigma_{0}$ be a complete $\sigma$-subalgebra of $\Sigma$. We also suppose that there is given a group of bimeasurable measure-preserving bijections $\left\{\theta_{\gamma}: \gamma \in \mathbb{R}\right\}$ on $\Omega$ such that $\theta_{\gamma}\left(\Sigma_{0}\right) \supset \Sigma_{0}$ for all $\gamma>0$. An example of such a group is given by the Wiener shifts satisfying the following condition:

$$
W(t+\gamma, \omega)-W(s+\gamma, \omega)=W\left(t, \theta_{\gamma}(\omega)\right)-W\left(s, \theta_{\gamma}(\omega)\right) \text { a.s. }
$$

for every real $t, s, \gamma($ see, for example, $[\mathbf{2}])$. Here and below $W_{t}:=W(t, \omega)$ is the standard Wiener process and $\Sigma_{0}$ is generated by random values $\{W(s, \omega): s \leqslant 0\}$. Consider a stochastic differential equation of Itô type in a phase space $\mathcal{B}$ (which is $\mathbb{R}^{n}$ for ordinary stochastic differential equations and a Banach space otherwise):

$$
\mathrm{d} x(t)=a(t, x(t)) \mathrm{d} t+b(t, x(t)) \mathrm{d} W_{t} .
$$

Assume that this equation has the unique (up to the natural almost sure equivalence) solution $x_{\alpha}(t)$ for each initial value $x(0)=\alpha \in L^{0}\left(\Omega, \Sigma_{0}, \mathbb{P} ; \mathcal{B}\right)$. This is for instance the case when $a$ and $b$ are Lipschitz-continuous functions. Assume in addition that $a$ and $b$ are $\tau$-periodic in time. Then the evolution (propagation) operator defined by $U(t, \alpha) x:=$ $x_{\alpha}(t)$ corresponding to equation (3.1) builds the so-called 'cocycle' (see, for example, [2]):

$$
T_{g} \circ U(t+\tau, \alpha)=U\left(t, T_{g} \alpha\right),
$$

where $T_{g}$ is the shift operator generated by the function $g:=\theta_{-\tau}$ according to the formula

$$
\left(T_{g} \xi\right)(\omega):=\xi(g(\omega))=\xi\left(\theta_{-\tau}(\omega)\right) .
$$

Now suppose we are looking for solutions of (3.1) periodic in distribution, i.e. satisfying

$$
\begin{aligned}
\mathbb{P}\left\{x\left(t_{1}\right) \in B_{1}, x\left(t_{2}\right)\right. & \left.\in B_{2}, \ldots, x\left(t_{k}\right) \in B_{k}\right\} \\
& =\mathbb{P}\left\{x\left(t_{1}+\tau\right) \in B_{1}, x\left(t_{2}+\tau\right) \in B_{2}, \ldots, x\left(t_{k}+\tau\right) \in B_{k}\right\}
\end{aligned}
$$

for every choice of the time points $t_{i} \in \mathbb{R}$, a number $k \in \mathbb{N}$ and Borel sets $B_{i} \subset \mathcal{B}$, $i=1, \ldots, k$, in the phase space of equation (3.1). In fact, it is the cocycle property that makes it hopeless to find a periodic solution in the usual sense: $U(t+\tau, \alpha)=U(t, \alpha)$ would typically imply that $T_{g} \circ U(\tau) \circ T_{g}^{-1}=U(\tau)$, which is not the case since $U$ is random (it essentially depends on $\omega$ ). We then have two reasonable notions of stochastically periodic solutions: strong and weak. While the latter is known to be equivalent to (3.3), 
the former is stronger. Both definitions are, however, acceptable for probabilists. For the sake of simplicity we consider only the strong periodic solution determined by the condition

$$
T_{g} x(t+\tau)=x(t) \quad \text { a.s. }
$$

The latter condition leads to the fixed-point problem

$$
\alpha=T(\alpha)
$$

with respect to the unknown $\alpha \in L^{0}\left(\Omega, \Sigma_{0}, \mathbb{P} ; \mathcal{B}\right)$, where $T:=T_{g} \circ U(\tau)$. Using the cocycle property (3.2) one can show that the solution of (3.1) starting at $\alpha$ is strongly periodic, if and only if it satisfies (3.4).

In [13] it has been shown that the operator $U(\tau)$ is local. Therefore, $T$ is atomic being a composition of a shift $T_{g}$ with the local operator. It is worth emphasizing that for ordinary stochastic differential equations, $U$ is usually generated by a Carathéodory function (see, for example, [2]), while even for simple delay equations it is not (see [12]).

Let us discuss now the relationship between disjointness-preserving operators called $D$-operators and atomic operators. For simplicity we restrict ourselves to the case of the operators $T: L^{0}(\Omega ; \mathbb{R}) \rightarrow L^{0}(\Omega ; \mathbb{R})$. Recall that $T$ is called a $D$-operator if it sends functions with disjoint supports into functions with disjoint supports, that is, for every $x, y \in L^{0}$, one has

$$
[\operatorname{supp} x] \cap[\operatorname{supp} y]=\tilde{\emptyset} \Rightarrow[\operatorname{supp} T(x)] \cap[\operatorname{supp} T(y)]=\tilde{\emptyset}
$$

where $\operatorname{supp} x:=\{\omega \in \Omega: x(\omega) \neq 0\}$ for $x \in L^{0}$. It is clear that every linear atomic operator is a $D$-operator. On the other hand, it is easy to prove using either the representation result due to von Neumann $[\mathbf{1 0}, \mathbf{1 8}]$ or the similar theorem of Sikorski [16, Theorem 32.3] that every linear continuous in measure $D$-operator $T: L^{0} \rightarrow L^{0}$ is a weighted shift $(T x)(\omega)=b(\omega) x(g(\omega))$ for some $\Sigma$-measurable function $b: \Omega \rightarrow \mathbb{R}$ and a $(\Sigma, \Sigma)$ measurable $g: \Omega \rightarrow \Omega$ satisfying

$$
\mu\left(g^{-1}(e)\right)=0 \quad \text { whenever } \mu(e)=0,
$$

and therefore is a linear atomic operator. An analogous result may be proven also when $\mathbb{R}$ is substituted by an arbitrary separable Banach space $\mathcal{X}$. However, such a perfect correspondence between atomic and $D$-operators is broken in the nonlinear case. In fact, in the following example we provide a nonlinear $D$-operator which is non-atomic.

Example 3.6. Let $\Omega:=\{0,1\}, \Sigma:=2^{\Omega}$ (the family of all subsets of $\Omega$ ), $\mu$ being any measure on $\Omega$ assigning a strictly positive value to each point, $\mathcal{X}:=\Omega$. Consider a function $h: \Omega \times \mathcal{X} \rightarrow \Omega$ such that $h(\omega, 0)=\omega$, but $h(\omega, 1) \neq \omega$ for all $\omega \in \Omega$.

Define the nonlinear shift operator $H: L^{0}(\Omega ; \mathcal{X}) \rightarrow L^{0}(\Omega ; \mathcal{X})$ by the relationship

$$
H(x)(\omega)=x(h(\omega, x)) .
$$


Clearly, $H$ is continuous in measure. To show that it is a $D$-operator, assume supp $x=e$, $\operatorname{supp} y \subset \Omega \backslash e$ and denote $h_{z}(\cdot):=h(\cdot, z(\cdot))$. Then

$$
\begin{aligned}
\operatorname{supp} H(x) & =\{\omega \in \Omega: h(\omega, x(\omega)) \in e\}=\left[(\Omega \backslash e) \cap h_{0}^{-1}(e)\right] \sqcup\left[e \cap h_{x}^{-1}(e)\right] \\
& =e \cap h_{x}^{-1}(e),
\end{aligned}
$$

since $h_{0}^{-1}(e)=e$. Similarly,

$$
\operatorname{supp} H(y) \subset\{\omega \in \Omega: h(\omega, y(\omega)) \in \Omega \backslash e\}=(\Omega \backslash e) \cap h_{y}^{-1}(\Omega \backslash e) .
$$

Thus, supp $H(x) \cap \operatorname{supp} H(y)=\emptyset$ and $H$ is a $D$-operator. On the other hand, taking $x=\mathbf{1}_{e}$ and $y \equiv 0$ we get $\{\omega \in \Omega: H(x)(\omega)=H(y)(\omega)\}=e$. If $H$ were atomic, the respective $\sigma$-homomorphism $F$ would have satisfied the relationship $F(e) \subset e$, so that necessarily $F(e)=e, h(\omega, x)=\omega$ and, hence, $H(x)=x$, which of course is not the case. Therefore, $H$ is not atomic.

This example explains why we could not directly use the definition of $D$-operators in the nonlinear situation, in spite of the fact that every atomic operator is evidently a $D$-operator. Otherwise, we would be forced to also cover in our theory nonlinear shifts, which usually have rather bad properties [7] and which therefore we wanted to avoid. On the other hand, our definition of atomic operators is equivalent to the definition of $D$-operators in the linear case, but it fits better into the nonlinear framework, as one can conclude from the results below.

\subsection{Representation}

We will prove that all the atomic operators acting between 'nice' spaces of measurable functions are actually the compositions of local operators and shifts. To begin with, we need the following auxiliary technical statement.

Lemma 3.7. Every $\sigma$-homomorphism $F: \Sigma_{1} \rightarrow \Sigma_{2}$ determines a unique linear continuous (in measure) operator $T_{F}: L^{0}\left(\Omega_{1} ; \mathcal{X}\right) \rightarrow L^{0}\left(\Omega_{2} ; \mathcal{X}\right)$, satisfying

$$
T_{F}\left(\mathbf{1}_{e_{1}} z\right)=\mathbf{1}_{F\left(e_{1}\right)} z
$$

for all $e_{1} \in \Sigma_{1}$ and $z \in \mathcal{X}$, where $\mathcal{X}$ is a separable Banach space. For this operator, called further generalized shift, one has $\operatorname{Im} T_{F}=L^{0}\left(\Omega_{2}, F \Sigma_{1} ; \mathcal{X}\right)$.

Proof.

Step 1. Define for all $z \in \mathcal{X}$ and for all $e_{1} \in \Sigma_{1}$ the value of the operator $T_{F}$ by means of (3.6), extend it by linearity to the set of all simple (i.e. finite-valued) functions in $L^{0}\left(\Omega_{1}, \Sigma_{1} ; \mathcal{X}\right)$, and then extend $T_{F}$ to the whole space $L^{0}\left(\Omega_{1}, \Sigma_{1} ; \mathcal{X}\right)$ by continuity (see [5, pp. 453, 454]). The uniqueness of the desired operator is immediate from this construction.

Step 2. Now we are to show that $\operatorname{Im} T_{F}=L^{0}\left(\Omega_{2}, F \Sigma_{1} ; \mathcal{X}\right)$. In fact, the inclusion

$$
\operatorname{Im} T_{F} \subset L^{0}\left(\Omega_{2}, F \Sigma_{1} ; \mathcal{X}\right)
$$


is obvious. To prove the reverse inclusion, take an arbitrary $y \in L^{0}\left(\Omega_{2}, F \Sigma_{1} ; \mathcal{X}\right)$, and represent it as a limit of a sequence of simple functions $\left\{y_{\nu}\right\} \subset L^{0}\left(\Omega_{2}, F \Sigma_{1} ; \mathcal{X}\right)$, i.e.

$$
y_{\nu}:=\sum_{i=1}^{N_{\nu}} \mathbf{1}_{F\left(e_{1}^{i, \nu}\right)} z_{i, \nu} \rightarrow y
$$

in measure $\mu_{2}$ on $\Omega_{2}$, where $z_{i, \nu} \in \mathcal{X}, e_{1}^{i, \nu} \in \Sigma_{1}$ and $N_{\nu} \in \mathbb{N}$. Then $y_{\nu}=T_{F} x_{\nu}$, where $x_{\nu}=\sum_{i=1}^{N_{\nu}} \mathbf{1}_{e_{1}^{i, \nu}} z_{i, \nu}$ are simple functions from $L^{0}\left(\Omega_{1}, \Sigma_{1} ; \mathcal{X}\right)$. Set

$$
E_{1}:=\left\{\omega_{1} \in \Omega_{1}: \frac{\mathrm{d} \mu_{F}}{\mathrm{~d} \mu_{1}}>0\right\}
$$

Letting $x_{\nu}^{\prime}=\sum_{i=1}^{N_{\nu}} \mathbf{1}_{e_{1}^{i, \nu} \cap E_{1}} z_{i, \nu}$ now, one observes immediately that $T_{F} x_{\nu}=T_{F} x_{\nu}^{\prime}$, but since $\left\{T_{F} x_{\nu}^{\prime}\right\}$ is a Cauchy sequence in $L^{0}\left(\Omega_{2}, F \Sigma_{1} ; \mathcal{X}\right)$, then so is $\left\{x_{\nu}^{\prime}\right\}$ in $L^{0}\left(\Omega_{1}, \Sigma_{1} ; \mathcal{X}\right)$, because $\mu_{F}$ is equivalent to $\mu_{1}$ over $E_{1}$. Therefore there is an $x \in L^{0}\left(\Omega_{1}, \Sigma_{1} ; \mathcal{X}\right)$ such that $x_{\nu}^{\prime} \rightarrow x$ in measure $\mu_{1}$ on $\Omega_{1}$, and, hence, $T_{F} x=y$, thus proving $\operatorname{Im} T_{F} \supset L^{0}\left(\Omega_{2}, F \Sigma_{1} ; \mathcal{X}\right)$ and showing the statement.

We may now proclaim the principal result of this subsection.

Theorem 3.8. Let $\mathcal{X}_{i}$ be separable Banach spaces. Then for every atomic operator $T: L^{0}\left(\Omega_{1} ; \mathcal{X}_{1}\right) \rightarrow L^{0}\left(\Omega_{2} ; \mathcal{X}_{2}\right)$ there is a local operator $N: L^{0}\left(\Omega_{2} ; \mathcal{X}_{1}\right) \rightarrow L^{0}\left(\Omega_{2} ; \mathcal{X}_{2}\right)$ such that

$$
T=N \circ T_{F} .
$$

The operator $T$ is continuous (in measure), if and only if the restriction of the operator $N$ to the subspace $L^{0}\left(\Omega_{2}, F \Sigma_{1} ; \mathcal{X}_{1}\right) \subset L^{0}\left(\Omega_{2}, \Sigma_{2} ; \mathcal{X}_{1}\right)$ is also continuous (in measure), where $F: \Sigma_{1} \rightarrow \Sigma_{2}$ is the $\sigma$-homomorphism corresponding to $T$. Moreover, if $\left(\Omega_{1}, \Sigma_{1}, \mu_{1}\right)$ is a standard measure space, then there is a $\left(\Sigma_{1}, \Sigma_{2}\right)$-measurable function $g: \Omega_{2} \rightarrow \Omega_{1}$ satisfying (2.2) such that $T=N \circ T_{g}$.

\section{Proof.}

Step 1. Define on $\operatorname{Im} T_{F}$ an operator $N: \operatorname{Im} T_{F} \rightarrow L^{0}\left(\Omega_{2}, \Sigma_{2}, \mu_{2} ; \mathcal{X}_{2}\right)$ by setting $N y:=T x$, if $y=T_{F} x$. First we show that the above definition of $N$ is correct. Assume that $y=T_{F} x$ and $y=T_{F} x^{\prime}$, while $x \neq x^{\prime}$. Let $\bar{x}:=x-x^{\prime}$ and $e_{1}^{\prime}:=\left\{\omega_{1} \in \Omega_{1}: \bar{x}\left(\omega_{1}\right)=\right.$ $0\}, e_{1}:=\Omega_{1} \backslash e_{1}^{\prime}$. We will prove that $\mu_{F}\left(e_{1}\right):=\mu_{2}\left(F\left(e_{1}\right)\right)=0$, hence $\tilde{F}\left(\tilde{e}_{1}^{\prime}\right)=\tilde{\Omega}_{2}$, and $T x=T x^{\prime}$, thus showing the correctness of the definition of $N$. In fact, if $\mu_{F}\left(e_{1}\right)>0$, then $\mu_{F}\left(e_{1} \cap E_{1}\right)>0$, where $E_{1} \subset \Omega_{1}$ is defined as in Step 2 of the proof of Lemma 3.7. Consider a sequence of simple functions $\left\{x_{\nu}\right\} \subset L^{0}\left(\Omega_{1}, \Sigma_{1}, \mu_{1} ; \mathcal{X}_{1}\right)$ with $\left.x_{\nu}\right|_{e_{1}^{\prime}}=0$ and $x_{\nu} \rightarrow \bar{x}$ in measure $\mu_{1}$ on $\Omega_{1}$, while $T_{F} x_{\nu} \rightarrow T_{F} \bar{x}=0$ in measure $\mu_{2}$ on $\Omega_{2}$. Since $T_{F}\left(\mathbf{1}_{E_{1}} x_{\nu}\right)=T_{F} x_{\nu}$ and $\mu_{F}$ is equivalent to $\mu_{1}$ over $E_{1}$, one has that $\mathbf{1}_{E_{1}} x_{\nu} \rightarrow 0$ in measure $\mu_{1}$ on $\Omega_{1}$, and, hence, $\mathbf{1}_{E_{1} \cap e_{1}} x=0$, contradicting the definition of $e_{1}$.

Step 2. We show that for all $e_{2} \in \Sigma_{2}$ from

$$
\left.y_{1}\right|_{e_{2}}=\left.y_{2}\right|_{e_{2}},
$$


where $\left\{y_{1}, y_{2}\right\} \subset L^{0}\left(\Omega_{2}, F \Sigma_{1}, \mu_{2} ; \mathcal{X}_{1}\right)$, it follows that

$$
\left.\left(N y_{1}\right)\right|_{e_{2}}=\left.\left(N y_{2}\right)\right|_{e_{2}} .
$$

For $e_{2} \in F \Sigma_{1}$ this is immediate, while if $e_{2} \notin F \Sigma_{1}$, then (3.8) implies the existence of $E_{2} \in F \Sigma_{1}, e_{2} \subset E_{2}$ such that $\left.y_{1}\right|_{E_{2}}=\left.y_{2}\right|_{E_{2}}$.

Step 3. We are now to extend $N$ over the whole $L^{0}\left(\Omega_{2}, \Sigma_{2}, \mu_{2} ; \mathcal{X}_{1}\right)$ as a local operator, i.e. so that (3.8) implies (3.9) for all $\left\{y_{1}, y_{2}\right\} \subset L^{0}\left(\Omega_{2}, \Sigma_{2}, \mu_{2} ; \mathcal{X}_{1}\right)$. Assume

$$
z \in L^{0}\left(\Omega_{2}, \Sigma_{2}, \mu_{2} ; \mathcal{X}_{1}\right) \backslash L^{0}\left(\Omega_{2}, F \Sigma_{1}, \mu_{2} ; \mathcal{X}_{1}\right) .
$$

Then, for every $e_{2} \in \Sigma_{2}$, there are two possibilities:

(i) either there is a $v \in L^{0}\left(\Omega_{2}, F \Sigma_{1} ; \mathcal{X}_{1}\right)$ which coincides with $z$ over $e_{2}$, or

(ii) there is no such $v \in L^{0}\left(\Omega_{2}, F \Sigma_{1} ; \mathcal{X}_{1}\right)$.

In case (i) we set $\left.(N z)\right|_{e_{2}}:=\left.(N v)\right|_{e_{2}}$, while in case (ii) $\left.(N z)\right|_{e_{2}}:=0$. This extension is well defined by Step 2 and is obviously local.

Step 4. Suppose that $T$ is continuous. By Lemma 3.7 the generalized shift $T_{F}$ is always continuous. Therefore the restriction of $N$ to $\operatorname{Im} T_{F}=L^{0}\left(\Omega_{2}, F \Sigma_{1} ; \mathcal{X}_{1}\right)$ must also be continuous.

Step 5. To prove the last claim, we observe that if $\left(\Omega_{1}, \Sigma_{1}, \mu_{1}\right)$ is a standard measure space, then by Theorem 32.3 of [16] there is a map $g: \Omega_{2} \rightarrow \Omega_{1}$ satisfying $g^{-1}\left(e_{1}\right)=$ $F\left(e_{1}\right)$ for all Borel sets $e_{1} \subset \Omega_{1}$. If $\Sigma_{1}$ also contains, besides Borel sets, some non-Borel $\mu_{1}$-nullsets, then

$$
\left[g^{-1}\left(e_{1}\right)\right]=\tilde{F}\left(\tilde{e}_{1}\right)
$$

easily follows from the above relationship. This implies that $g$ is $\left(\Sigma_{1}, \Sigma_{2}\right)$-measurable and satisfies $(2.2)$, while $T_{g} x=T_{F} x$ for every simple $x \in L^{0}\left(\Omega_{1} ; \mathcal{X}_{1}\right)$ and, hence, by continuity, everywhere over this space.

Corollary 3.9. Let $\left(\Omega_{1}, \Sigma_{1}, \mu_{1}\right)$ be a standard measure space, $\mathcal{X}_{i}$ be separable metric spaces and let $T: L^{0}\left(\Omega_{1} ; \mathcal{X}_{1}\right) \rightarrow L^{0}\left(\Omega_{2} ; \mathcal{X}_{2}\right)$ be an atomic operator. Under the continuum hypothesis there is a measurable function $g: \Omega_{2} \rightarrow \Omega_{1}$ satisfying (2.2) and a function $f: \Omega_{2} \times \mathcal{X}_{1} \rightarrow \mathcal{X}_{2}$ such that $T=N_{f} \circ T_{g}$, i.e. for all $x \in L^{0}\left(\Omega_{1} ; \mathcal{X}_{1}\right)$ one has

$$
(T x)\left(\omega_{2}\right)=f\left(\omega_{2}, x\left(g\left(\omega_{2}\right)\right)\right) \quad \mu_{2} \text {-a.e. on } \Omega_{2} .
$$

Proof. It is enough to apply Theorem 3.8 using the representation of every local operator as a Nemytskiǐ operator provided in [11] (see also [1]).

This corollary justifies our terminology: we may now think of an atomic operator as a kind of integral operator with respect to the random atomic measure concentrated in the point $g\left(\omega_{2}\right)$. A similar terminology is widely used in the linear case (see, for example, $[\mathbf{2 0}])$. 
We note that even if $T: L^{0}\left(\Omega_{1} ; \mathcal{X}_{1}\right) \rightarrow L^{0}\left(\Omega_{2} ; \mathcal{X}_{2}\right)$ is continuous, the operator $N$ defined in Theorem 3.8 need not be continuous (only its restriction to a certain subspace is). Therefore, even in the situation of Corollary 3.9, the function $f: \Omega_{2} \times \mathcal{X}_{1} \rightarrow \mathcal{X}_{2}$, generating together with $g: \Omega_{2} \rightarrow \Omega_{1}$ the operator $T$, need not be a Carathéodory function. An example from the theory of stochastic processes, which we are going to discuss now, shows that there are indeed atomic operators $T$ not representable by a composition of a Nemytskiǐ operator generated by a Carathéodory function and a shift operator.

Example 3.10. Consider as in Example 3.5 a probability space $(\Omega, \Sigma, \mathbb{P})$, the standard Wiener process $W_{t}$, the Wiener shift $g:=\theta_{-1}: \Omega \rightarrow \Omega$ inducing the isomorphism of the $\sigma$-subalgebras $\Sigma_{0}$ and $\Sigma_{1}:=g^{-1}\left(\Sigma_{0}\right)$. Letting $\mathcal{X}:=L^{2}[0,1]$, define the operator $T: L^{0}\left(\Omega, \Sigma_{1}, \mathbb{P} ; \mathcal{X}\right) \rightarrow L^{0}\left(\Omega, \Sigma_{1}, \mathbb{P} ; \mathcal{X}\right)$ as the stochastic integration with respect to the Wiener process

$$
(T x)(\omega):=\int_{0}^{(\cdot)} x(s, g(\omega)) \mathrm{d} W_{s}(\omega) .
$$

Note that we shifted the $\Sigma_{1}$-measurable integrand $x(t, \omega)$ with the help of $g$. In this way the stochastic process $x(s, g(\omega))$ becomes $\Sigma_{0}$-measurable, so that the stochastic integral is well defined. The operator $T$ is atomic since it is a composition of the stochastic integral (which is local) and the shift $T_{g}$. However, the stochastic integral cannot be represented by a Nemytskiĩ operator generated by a Carathéodory function. Otherwise, the stochastic integral could have been, by the Riesz representation theorem, reduced to the ordinary Lebesgue-Stieltjes integral, which is impossible (see, for example, [13] or [14]).

\subsection{Acting, continuity and boundedness}

We suppose throughout this subsection that $\left(\Omega_{2}, \Sigma_{2}, \mu_{2}\right)$ supports a non-atomic measure and we let both $\mathcal{X}_{1}$ and $\mathcal{X}_{2}$ be separable Banach spaces.

Theorem 3.11. If a continuous-in-measure atomic operator $T: L^{0}\left(\Omega_{1} ; \mathcal{X}_{1}\right) \rightarrow$ $L^{0}\left(\Omega_{2} ; \mathcal{X}_{2}\right)$ continuously maps $L^{p}\left(\Omega_{1} ; \mathcal{X}_{1}\right)$ into $L^{q}\left(\Omega_{2} ; \mathcal{X}_{2}\right)$, where $1 \leqslant p, q<\infty$, then $T$ sends bounded sets of $L^{p}$ into bounded sets of $L^{q}$.

\section{Proof.}

Step 1. According to the representation theorem 3.8, the continuity in measure of the operator $T$ is equivalent to that of the restriction of the local operator $N$ in the representation formula (3.7) to the space $L^{0}\left(\Omega_{2}, F \Sigma_{1}, \mu_{2} ; \mathcal{X}_{1}\right)$, where $F: \Sigma_{1} \rightarrow \Sigma_{2}$ is the respective $\sigma$-homomorphism making $T$ atomic. We then make the following auxiliary construction. Suppose without loss of generality $T(0)=0$. Let $\mu_{F}\left(e_{1}\right):=\mu_{2}\left(F\left(e_{1}\right)\right)$, observe that $\mu_{F} \ll \mu_{1}$ and consider the set

$$
E_{1}:=\left\{\omega_{1} \in \Omega_{1}: \frac{\mathrm{d} \mu_{F}}{\mathrm{~d} \mu_{1}}>0\right\} .
$$

To show the statement, it is enough to prove, that if $T: L^{p}\left(\Omega_{1} ; \mathcal{X}_{1}\right) \rightarrow L^{q}\left(\Omega_{2} ; \mathcal{X}_{2}\right)$ is continuous, then its restriction to $L^{p}\left(E_{1} ; \mathcal{X}_{1}\right)$ is bounded. In fact, this follows from $T x=N\left(T_{F} x\right)=N\left(T_{F}\left(\mathbf{1}_{E_{1}} x\right)\right)$. 
Step 2. Consider the operator $P$ defined formally by $(P x)\left(\omega_{2}\right):=\left|(N x)\left(\omega_{2}\right)\right|_{\mathcal{X}_{2}}^{q}$. Note that since

$$
\int_{\Omega_{2}}\left|\left(T_{F} x\right)\left(\omega_{2}\right)\right|_{\mathcal{X}_{1}}^{p} \mathrm{~d} \mu_{2}\left(\omega_{2}\right)=\int_{\Omega_{1}} \frac{\mathrm{d} \mu_{F}}{\mathrm{~d} \mu_{1}}\left|x\left(\omega_{1}\right)\right|_{\mathcal{X}_{1}}^{p} \mathrm{~d} \mu_{1}\left(\omega_{1}\right)
$$

and $T_{F}(x \cdot y)=\left(T_{F} x\right) \cdot\left(T_{F} y\right)$, then

$$
\int_{E_{1}}\left|x\left(\omega_{1}\right)\right|_{\mathcal{X}_{1}}^{p} \mathrm{~d} \mu_{1}\left(\omega_{1}\right)=\int_{F\left(E_{1}\right)} T_{F}\left(\frac{\mathrm{d} \mu_{F}}{\mathrm{~d} \mu_{1}}\right)^{-1}\left|\left(T_{F} x\right)\left(\omega_{2}\right)\right|_{\mathcal{X}_{1}}^{p} \mathrm{~d} \mu_{2}\left(\omega_{2}\right),
$$

and, hence, $T_{F}$ is an isometry between $L^{p}\left(E_{1}, \mu_{1} ; \mathcal{X}_{1}\right)$ and $L^{p}\left(F\left(E_{1}\right), \mu_{2}^{\prime} ; \mathcal{X}_{1}\right)$, where

$$
\mu_{2}^{\prime}=T_{F}\left(\frac{\mathrm{d} \mu_{F}}{\mathrm{~d} \mu_{1}}\right)^{-1} \mu_{2}
$$

Therefore, if $T: L^{p}\left(E_{1}, \Sigma_{1}, \mu_{1} ; \mathcal{X}_{1}\right) \rightarrow L^{q}\left(\Omega_{2}, \Sigma_{2}, \mu_{2} ; \mathcal{X}_{2}\right)$ is continuous, then

$$
P: L^{p}\left(F\left(E_{1}\right), F\left(\Sigma_{1} \cap E_{1}\right), \mu_{2}^{\prime} ; \mathcal{X}_{1}\right) \rightarrow L^{1}\left(F\left(E_{1}\right), \Sigma_{2}, \mu_{2} ; \mathbb{R}\right)
$$

is also continuous. Hence, so is the operator

$$
Q: L^{p}\left(F\left(E_{1}\right), F\left(\Sigma_{1} \cap E_{1}\right), \mu_{2}^{\prime} ; \mathcal{X}_{1}\right) \rightarrow L^{1}\left(F\left(E_{1}\right), \Sigma_{2}, \mu_{2}^{\prime} ; \mathbb{R}\right)
$$

defined by

$$
(Q x)\left(\omega_{2}\right):=\left(T_{F} \frac{\mathrm{d} \mu_{F}}{\mathrm{~d} \mu_{1}}\right)\left(\omega_{2}\right)(P x)\left(\omega_{2}\right)=\left(T_{F} \frac{\mathrm{d} \mu_{F}}{\mathrm{~d} \mu_{1}}\right)\left(\omega_{2}\right)\left|(N x)\left(\omega_{2}\right)\right|_{\mathcal{X}_{2}}^{q} .
$$

Moreover, both $P$ and $Q$ are clearly the restrictions of some local operators.

Step 3. Now let $\mathcal{E}$ stand for the conditional expectation with respect to the $\sigma$ subalgebra $F\left(\Sigma_{1} \cap E_{1}\right)$ (see, for example, Chapter XI of [6] or Example 4.6 below). Since $\mathcal{E}\left(\mathbf{1}_{e} x\right)=\mathbf{1}_{e} \mathcal{E}(x)$ for all $x \in L^{1}\left(\Omega_{2}, \Sigma_{2}, \mu_{2} ; \mathbb{R}\right)$ and for arbitrary $e \in F\left(\Sigma_{1} \cap E_{1}\right)$, the composition

$$
\mathcal{E} \circ Q: L^{p}\left(F\left(E_{1}\right), F\left(\Sigma_{1} \cap E_{1}\right), \mu_{2}^{\prime} ; \mathcal{X}_{1}\right) \rightarrow L^{q}\left(F\left(E_{1}\right), F\left(\Sigma_{1} \cap E_{1}\right), \mu_{2}^{\prime} ; \mathbb{R}\right)
$$

is a continuous local operator. Now both images and preimages of $\mathcal{E} \circ Q$ 'live' in the same measure space and we may apply the second representation result from [13], which provides a Carathéodory function $\varphi: \Omega_{2} \times \mathcal{X}_{1} \rightarrow \mathbb{R}$, for which

$$
(\mathcal{E} \circ Q)(x)\left(\omega_{2}\right)=\varphi\left(\omega_{2}, x\left(\omega_{2}\right)\right) \quad \mu_{2}^{\prime} \text {-a.e. on } F\left(E_{1}\right) .
$$

Choose an arbitrary bounded $B \subset L^{p}\left(E_{1}, \Sigma_{1}, \mu_{1} ; \mathcal{X}_{1}\right)$. Then the set $T_{F} B$ is bounded in $L^{p}\left(F\left(E_{1}\right), F\left(\Sigma_{1} \cap E_{1}\right), \mu_{2}^{\prime} ; \mathcal{X}_{1}\right)$. Since for Nemytskiǐ operators generated by Carathéodory functions, continuity and boundedness in Lebesgue spaces are equivalent [1, Theorems 3.1 and 3.2], then

$$
(\mathcal{E} \circ Q)\left(T_{F} B\right) \subset L^{1}\left(F\left(E_{1}\right), F\left(\Sigma_{1} \cap E_{1}\right), \mu_{2}^{\prime} ; \mathbb{R}\right)
$$


is also bounded. But

$$
\begin{aligned}
\|(\mathcal{E} \circ Q)(x)\|_{1} & =\int_{F\left(E_{1}\right)}(\mathcal{E} \circ Q)(x)\left(\omega_{2}\right) \mathrm{d} \mu_{2}^{\prime}\left(\omega_{2}\right) \\
& =\int_{F\left(E_{1}\right)} Q(x)\left(\omega_{2}\right) \mathrm{d} \mu_{2}^{\prime}\left(\omega_{2}\right)=\|Q(x)\|_{1}
\end{aligned}
$$

by the property of conditional expectation, where $\|\cdot\|_{1}$ stands for the norm in $L^{1}\left(F\left(E_{1}\right)\right.$, $\left.\Sigma_{1} \cap E_{1}, \mu_{2}^{\prime} ; \mathbb{R}\right)$. Thus $Q\left(T_{F} B\right)$ is bounded in $L^{1}\left(F\left(E_{1}\right), \Sigma_{1} \cap E_{1}, \mu_{2}^{\prime} ; \mathbb{R}\right)$, from which it also follows that $P\left(T_{F} B\right)$ is bounded in $L^{1}\left(F\left(E_{1}\right), \Sigma_{1} \cap E_{1}, \mu_{2} ; \mathbb{R}\right)$, proving the statement.

The mere fact that the atomic operator maps one Lebesgue space into another does not mean that it is continuous, since even boundedness does not imply continuity for such operators, in contrast to the case of Nemytskiĩ operators, where boundedness and continuity are often equivalent.

Example 3.12. Let $\Omega_{1}:=(0,1)$ and $\Omega_{2}:=(0,1) \times(0,1), \mu_{1}$ and $\mu_{2}$ be one- and twodimensional Lebesgue measures in $\Omega_{1}$ and $\Omega_{2}$, respectively, while $\Sigma_{1}$ and $\Sigma_{2}$ stand for the respective Lebesgue $\sigma$-algebras. We will construct an atomic operator $T: L^{p}\left(\Omega_{1} ; \mathbb{R}\right) \rightarrow$ $L^{p}\left(\Omega_{2} ; \mathbb{R}\right)$, which is bounded and continuous in measure, but is discontinuous as an operator between the mentioned Lebesgue spaces.

For this purpose choose a sequence $\left\{\alpha_{\nu}\right\}$ in the unit ball $B \subset L^{p}(0,1)$ converging to zero in measure, but not in the norm of $L^{p}(0,1)$. Without loss of generality we may assume that the sequence consists of different members. Set $\gamma(1 / \nu):=\alpha_{\nu}$ for $\nu \in \mathbb{N}$ and extend $\gamma$ to be a measure-continuous function $\gamma: \mathbb{R} \rightarrow B$. The latter extension can be obtained by first setting

$$
\gamma((1-\tau) / \nu+\tau /(\nu+1)):=(1-\tau) \alpha_{\nu}+\tau \alpha_{\nu+1}
$$

for $0 \leqslant \tau \leqslant 1$, which gives a measure-continuous function $\gamma:(0,1) \rightarrow B$ and then extending the latter to the whole $\mathbb{R}$ by means of a projection $v: \mathbb{R} \rightarrow(0,1)$. By construction, the function $\gamma$ is discontinuous with respect to the norm topology of $L^{p}(0,1)$, but it is bounded in the space $L^{p}(0,1)$.

Note that $L^{p}\left(\Omega_{2} ; \mathbb{R}\right)$ may be naturally identified with $L^{p}\left(\Omega_{1} ; L^{p}(0,1)\right)$. In fact, if $\omega_{2} \in$ $\Omega_{2}$ is represented as $\omega_{2}=(t, s)$, where $t, s \in \Omega_{1}$, then every element $z \in L^{p}\left(\Omega_{2} ; \mathbb{R}\right)$ may be viewed as a function $z: \Omega_{1} \rightarrow L^{p}(0,1)$ which maps $t \in \Omega_{1} \mapsto z(t, \cdot) \in L^{p}(0,1)$ and belongs to $L^{p}\left(\Omega_{1} ; L^{p}(0,1)\right)$. We now set

$$
T(x)\left(\omega_{2}\right) \equiv T(x)(t, s):=\gamma(x(t))(s),
$$

where $x \in L^{p}\left(\Omega_{1} ; \mathbb{R}\right)$. Clearly, $T$ is continuous in measure since so is $\gamma$. Moreover, $T$ sends bounded sets of $L^{p}\left(\Omega_{1} ; \mathbb{R}\right)$ into bounded sets of $L^{p}\left(\Omega_{2} ; \mathbb{R}\right)$, for

$$
\|T x\|^{p}=\int_{0}^{1} \mathrm{~d} t \int_{0}^{1}|(T x)(t, s)|^{p} \mathrm{~d} s=\int_{0}^{1}\|\gamma(x(t))\|_{L^{p}(0,1)}^{p} \mathrm{~d} t \leqslant 1 .
$$

This operator is, however, discontinuous as an operator between the mentioned Lebesgue spaces, because the sequence $\{T(1 / \nu)\}$ does not converge in $L^{p}(0,1)$. Finally, it remains 
to note that $T$ is atomic with respect to the natural embedding $F: \Sigma_{1} \rightarrow \Sigma_{2}$ defined by $F\left(e_{1}\right):=e_{1} \times(0,1)$. Indeed, if $x(t)=y(t)$ for a.e. $t \in e_{1} \in \Sigma_{1}$, then

$$
T(x)\left(\omega_{2}\right)=\gamma(x(t))(s)=\gamma(y(t))(s)=T(y)\left(\omega_{2}\right) \quad \mu_{2} \text {-a.e. on } F\left(e_{1}\right) .
$$

\subsection{Non-compactness}

The atomic operators acting in the spaces of measurable functions possess, much like the local operators, an important property of non-compactness.

Theorem 3.13. Assume $0 \leqslant p, q \leqslant \infty$ and $\mathcal{X}_{i}$ are separable Banach spaces. Then a non-constant atomic operator $T: L^{p}\left(\Omega_{1} ; \mathcal{X}_{1}\right) \rightarrow L^{q}\left(\Omega_{2} ; \mathcal{X}_{2}\right)$ is not compact, provided $\mu_{1}$ is a non-atomic measure.

Proof. Since $T$ is non-constant, there exists an $x \in L^{p}\left(\Omega_{1} ; \mathcal{X}_{1}\right)$ and $E_{2} \in \Sigma_{2}, \mu_{2}\left(E_{2}\right)>$ 0 , such that $(T(x))\left(\omega_{2}\right) \neq(T(0))\left(\omega_{2}\right)$ for all $\omega_{2} \in E_{2}$. Without loss of generality we may suppose $T(0)=0$ (a function $T(0)$, which can always be added, does not make any influence on the compactness properties of the operator). Define then $\hat{\mu}_{F}\left(e_{1}\right):=$ $\mu_{2}\left(F\left(e_{1}\right) \cap E_{2}\right)$. Clearly, $\hat{\mu}_{F}$ is a measure on $\Sigma_{1}$, while $\hat{\mu}_{F} \ll \mu_{1}$ and, hence, is nonatomic. Note that $\hat{\mu}_{F}\left(\Omega_{1}\right)=\mu_{2}\left(E_{2}\right)$.

We construct partitions of $\Omega_{1}$ by the following inductive process. First find such $\Omega_{1}^{0} \in$ $\Sigma_{1}, \Omega_{1}^{1} \in \Sigma_{1}$, that $\Omega_{1}:=\Omega_{1}^{0} \sqcup \Omega_{1}^{1}$ with

$$
\hat{\mu}_{F}\left(\Omega_{1}^{0}\right)=\hat{\mu}_{F}\left(\Omega_{1}^{1}\right)=\mu_{2}\left(E_{2}\right) / 2 .
$$

On the $k$ th step, having constructed a partition of $\Omega_{1}$ into sets $\Omega_{1}^{i_{1} \ldots i_{k}}, i_{j} \in\{0,1\}$, such that

$$
\Omega_{1}=\bigsqcup_{\substack{i_{j} \in\{0,1\} \\ j=1, \ldots, k}} \Omega_{1}^{i_{1} \ldots i_{k}}, \quad \hat{\mu}_{F}\left(\Omega_{1}^{i_{1} \ldots i_{k}}\right)=\frac{1}{2^{k}} \mu_{2}\left(E_{2}\right),
$$

we divide each of the sets $\Omega_{1}^{i_{1} \ldots i_{k}}$ into two, so that

$$
\Omega_{1}^{i_{1} \ldots i_{k}}=\Omega_{1}^{i_{1} \ldots i_{k} 0} \bigsqcup \Omega_{1}^{i_{1} \ldots i_{k} 1}, \quad \hat{\mu}_{F}\left(\Omega_{1}^{i_{1} \ldots i_{k} 0}\right)=\hat{\mu}_{F}\left(\Omega_{1}^{i_{1} \ldots i_{k} 1}\right)=\frac{1}{2^{k+1}} \mu_{2}\left(E_{2}\right) .
$$

Now we set

$$
E_{1}(k):=\bigcup_{\substack{i_{j} \in\{0,1\} \\ j=1, \ldots, k}} \Omega_{1}^{i_{1} \ldots i_{k} 0}
$$

observing that $\hat{\mu}_{F}\left(E_{1}(k)\right)=\mu_{2}\left(E_{2}\right) / 2$ for all $k \in \mathbb{N}$. The sequence of functions $x_{k}:=$ $\mathbf{1}_{E_{1}(k)} x$ is bounded in $L^{p}\left(\Omega_{1} ; \mathcal{X}_{1}\right)$, but the sequence of images $T\left(x_{k}\right)$ is not precompact in $L^{q}\left(\Omega_{2} ; \mathcal{X}_{2}\right)$, because

$$
\left|\left(T x_{j}\right)\left(\omega_{2}\right)-\left(T x_{k}\right)\left(\omega_{2}\right)\right|_{\mathcal{X}_{2}}=\mathbf{1}_{F\left(E_{1}(j)\right) \Delta F\left(E_{1}(k)\right)}|T(x)|_{\mathcal{X}_{2}},
$$

while for $j<k$ the latter is equal to $|T(x)| \mathcal{X}_{2}>0$ on the set

$$
E_{2}(j, k):=F\left(E_{1}(j) \Delta E_{1}(k)\right)=F\left(\bigcup_{i_{j} \neq i_{k}} \Omega_{1}^{i_{1} \ldots i_{j} \ldots i_{k}}\right)
$$

of the measure $\mu_{2}\left(E_{2}(j, k)\right) \geqslant \hat{\mu}_{F}\left(E_{1}(j) \Delta E_{1}(k)\right)=\mu_{2}\left(E_{2}\right) / 2>0$. 
The proof of Theorem 3.13 extends the well-known proof of non-compactness of the Nemytskiǐ operator (see Theorem 1.8 of [1] ), its main idea going back to Krasnosel'skiǐ. We remark also that the same argument gives non-compactness of the atomic operators in more general ideal Banach spaces.

\subsection{Weak continuity}

Our next result extends the well-known theorem on the degeneracy of the Nemytskii operator in the weak topologies of Lebesgue spaces [1, Theorem 2.10] to the case of atomic operators.

Theorem 3.14. Let $1 \leqslant p, q<\infty, \mu_{1}$ be a non-atomic measure and $\mathcal{X}_{i}$ be separable Banach spaces. Then an atomic operator $T: L^{p}\left(\Omega_{1} ; \mathcal{X}_{1}\right) \rightarrow L^{q}\left(\Omega_{2} ; \mathcal{X}_{2}\right)$ is weakly continuous, if and only if $T$ is affine, i.e. $T(\cdot)-T(0)$ is a linear bounded operator.

Proof. The only non-trivial part of the statement is the 'only if' part. To prove this part, it clearly suffices to show that

$$
T(\lambda x+(1-\lambda) y)=\lambda T(x)+(1-\lambda) T(y)
$$

for all $\lambda \in[0,1]$ and $x, y \in L^{p}\left(\Omega_{1} ; \mathcal{X}_{1}\right)$. Given such a $\lambda$, we may, since $\mu_{1}$ is non-atomic, choose a sequence of sets $\left\{e_{1}^{\nu}\right\} \subset \Sigma_{1}$ such that $\mathbf{1}_{e_{1}^{\nu}} \rightarrow \lambda$ *weakly in $L^{\infty}\left(\Omega_{1} ; \mathbb{R}\right)$. Then also $\mathbf{1}_{F\left(e_{1}^{\nu}\right)}=T_{F} \mathbf{1}_{e_{1}^{\nu}} \rightarrow \lambda$ *-weakly in $L^{\infty}\left(\Omega_{2} ; \mathbb{R}\right)$, where $F: \Sigma_{1} \rightarrow \Sigma_{2}$ is the $\sigma$-homomorphism making $T$ atomic, and, hence,

$$
\mathbf{1}_{e_{1}^{\nu}} x+\mathbf{1}_{\Omega_{1} \backslash e_{1}^{\nu}} y \rightarrow \lambda x+(1-\lambda) y \quad \text { *-weakly in } L^{\infty}\left(\Omega_{1} ; \mathcal{X}_{1}\right) .
$$

On the other hand, supposing without loss of generality that $T(0)=0$, one has $T\left(\mathbf{1}_{e_{1}} x\right)=$ $\mathbf{1}_{F\left(e_{1}\right)} T(x)$ for every $x \in L^{p}\left(\Omega_{1} ; \mathcal{X}_{1}\right)$ and $e_{1} \in \Sigma_{1}$. Therefore,

$$
T\left(\mathbf{1}_{e_{1}^{\nu}} x+\mathbf{1}_{\Omega_{1} \backslash e_{1}^{\nu}} y\right) \rightarrow \lambda T(x)+(1-\lambda) T(y) \quad \text { *-weakly in } L^{\infty}\left(\Omega_{2} ; \mathcal{X}_{2}\right) .
$$

Combining now (3.11) and (3.12) with weak continuity of $T$, one obtains (3.10), thus concluding the proof.

\subsection{Convergence}

In [9] it has been proven (though in a bit more general terminology) that the strong (pointwise) limit of a sequence of shift operators in Lebesgue spaces is still a shift operator. In [8] this assertion was generalized for weighted shifts. This corresponds well to the theorem below which asserts the atomicity of the strong (pointwise) limits of general atomic operators.

Theorem 3.15. Assume that $0<p<\infty, 0 \leqslant q \leqslant \infty, \Sigma_{1}$ is countably generated and $\mathcal{X}_{i}, i=1,2$, are separable Banach spaces. Let a sequence of atomic operators $T_{\nu}$ : $L^{p}\left(\Omega_{1} ; \mathcal{X}_{1}\right) \rightarrow L^{q}\left(\Omega_{2} ; \mathcal{X}_{2}\right)$, converge strongly (pointwise) to an operator $T$, which maps $L^{p}$-convergent sequences into measure convergent ones. Then $T$ is atomic. 
Proof.

Step 1. Before starting the proof we make some necessary arrangements. Firstly, observe that since $L^{q}\left(\Omega_{2} ; \mathcal{X}_{2}\right) \hookrightarrow L^{0}\left(\Omega_{2} ; \mathcal{X}_{2}\right)$, we may assume without loss of generality that $q=0$. Secondly, we may let all the operators satisfy $T_{\nu}(0)=0$, hence also $T(0)=0$. Finally, note that due to our assumption on $\Sigma_{1}$, the space $L^{p}\left(\Omega_{1}, \Sigma_{1}, \mu_{1} ; \mathcal{X}_{1}\right)$ is separable, so that we can choose its countable dense subset $\left\{z_{i}\right\}$. Now we set

$$
A_{i}:=\left\{\omega_{2} \in \Omega_{2}: T\left(z_{i}\right)\left(\omega_{2}\right) \neq 0\right\}, \quad E_{2}:=\bigcup_{i=1}^{\infty} A_{i}, \quad B_{1}:=A_{1}, \quad B_{i}:=A_{i} \backslash \bigcup_{j=1}^{i-1} B_{j},
$$

Without loss of generality we may suppose that $\mu_{2}\left(B_{i}\right)>0$ for all $i \in \mathbb{N}$ (otherwise one just renumbers the sequence throwing out the nullsets). Clearly, $T(x)\left(\omega_{2}\right)=0$ for $\mu_{2}$-a.e. $\omega_{2} \in \Omega_{2} \backslash E_{2}$ and for every $x \in L^{p}\left(\Omega_{1} ; \mathcal{X}_{1}\right)$.

Step 2. Assume that $F_{\nu}: \Sigma_{1} \rightarrow \Sigma_{2}$ are the $\sigma$-homomorphisms making the respective $T_{\nu}$ atomic. We construct the 'limit' $\sigma$-homomorphism $F$ to be used in the proof. For this purpose define the operators $\Gamma_{i, \nu}: L^{p}\left(\Omega_{1} ; \mathcal{X}_{1}\right) \rightarrow L^{0}\left(\Omega_{2} ; \overline{\mathbb{R}}\right)$, where $\overline{\mathbb{R}}:=\mathbb{R} \cup\{+\infty\}$, by the formula

$$
\Gamma_{i, \nu}(x)\left(\omega_{2}\right):=\frac{\left|T_{\nu}(x)\left(\omega_{2}\right)\right|_{\mathcal{X}_{2}}}{\left|T_{\nu}\left(z_{i}\right)\left(\omega_{2}\right)\right| \mathbf{X}_{2}} \mathbf{1}_{B_{i}}\left(\omega_{2}\right)
$$

Observe that the denominators may vanish for some $\omega_{2}$ so that images of the operators $\Gamma_{i, \nu}$ admit infinite values. Therefore, for technical reasons we assume that $0 / 0=0$ and $a / 0=+\infty$, if $a \neq 0$. Clearly, $\Gamma_{i, \nu}$ are atomic operators and converge strongly (pointwise) as $\nu \rightarrow \infty$ to the operator $\Gamma_{i}: L^{p}\left(\Omega_{1} ; \mathcal{X}_{1}\right) \rightarrow L^{0}\left(\Omega_{2} ; \overline{\mathbb{R}}\right)$ given by the formula

$$
\Gamma_{i}(x)\left(\omega_{2}\right):=\frac{\left|T(x)\left(\omega_{2}\right)\right| \mathcal{X}_{2}}{\left|T\left(z_{i}\right)\left(\omega_{2}\right)\right| \mathcal{X}_{2}} \mathbf{1}_{B_{i}}\left(\omega_{2}\right) .
$$

For an arbitrary $e_{1} \in \Sigma_{1}$ we take $x_{i}:=\mathbf{1}_{e_{1}} z_{i}$ and observe that

$$
\Gamma_{i, \nu}\left(x_{i}\right)=\mathbf{1}_{F_{\nu}\left(e_{1}\right) \cap A_{i}^{\nu} \cap B_{i}},
$$

where

$$
A_{i}^{\nu}:=\left\{\omega_{2} \in \Omega_{2}: T_{\nu}\left(z_{i}\right)\left(\omega_{2}\right) \neq 0\right\} .
$$

Since, up to a subsequence, $\mathbf{1}_{A_{i}^{\nu}} \rightarrow \mathbf{1}_{A_{i}} \mu_{2}$-a.e. on $\Omega_{2}$ and $\Gamma_{i, \nu}\left(x_{i}\right) \rightarrow \Gamma_{i}\left(x_{i}\right)$ in measure $\mu_{2}$ on $\Omega_{2}$, as $\nu \rightarrow \infty$, then also $\left\{\mathbf{1}_{F_{\nu}\left(e_{1}\right) \cap B_{i}}\right\}$ converges in measure $\mu_{2}$ on $\Omega_{2}$. The limit is again a characteristic function of some set which we denote by $\Psi_{i}\left(e_{1}\right)$. Note that $\Psi_{i}\left(e_{1}\right) \subset B_{i}$ by construction. Moreover, each $\Psi_{i}: \Sigma_{1} \rightarrow \Sigma_{2} \cap B_{i}$ is a $\sigma$-homomorphism, since so are all the maps $e_{1} \mapsto F_{\nu}\left(e_{1}\right) \cap B_{i}$. To show that $\Psi_{i}$ preserves nullsets, it is enough to apply the Vitaly-Hahn-Saks Theorem (see $\S$ IX.10 of [6]) to the sequence of measures $\left\{\mu_{1}^{\nu}\right\}$ over $\Sigma_{1}$ defined by $\mu_{1}^{\nu}\left(e_{1}\right):=\mu_{2}\left(F_{\nu}\left(e_{1}\right) \cap B_{i}\right)$. In fact, according to this theorem the measure $\mu_{1}^{\infty}$ defined by $\mu_{1}^{\infty}\left(e_{1}\right):=\lim _{\nu} \mu_{1}^{\nu}\left(e_{1}\right)=\mu_{2}\left(\Psi_{i}\left(e_{1}\right)\right)$ is absolutely continuous with respect to $\mu_{1}$, since so are all $\mu_{1}^{\nu}$. 
Now, noting that each of $B_{i}$ is disjoint with $\Omega_{2} \backslash E_{2}$, we define a map $F: \Sigma_{1} \rightarrow \Sigma_{2}$ by

$$
F\left(e_{1}\right):=\left(\bigsqcup_{i=1}^{\infty} \Psi_{i}\left(e_{1}\right)\right) \bigsqcup\left(F_{1}\left(e_{1}\right) \backslash E_{2}\right) .
$$

Clearly, $F$ is a $\sigma$-homomorphism from $\Sigma_{1}$ to $\Sigma_{2}$. It is also obvious that for every $e_{1} \in \Sigma_{1}$ we have $\mathbf{1}_{F_{\nu}\left(e_{1}\right) \cap E_{2}} \rightarrow \mathbf{1}_{F\left(e_{1}\right) \cap E_{2}}$ in measure as $\nu \rightarrow \infty$.

Step 3. Observe now that if $\left.x\right|_{e_{1}}=\left.y\right|_{e_{1}}$, then

$$
\mathbf{1}_{E_{2}} T\left(\mathbf{1}_{e_{1}} x\right)=\lim _{\nu \rightarrow \infty} \mathbf{1}_{E_{2}} T_{\nu}\left(\mathbf{1}_{e_{1}} x\right)=\lim _{\nu \rightarrow \infty} \mathbf{1}_{F_{\nu}\left(e_{1}\right) \cap E_{2}} T_{\nu}(x)=\mathbf{1}_{F\left(e_{1}\right) \cap E_{2}} T(x)
$$

and, analogously, $\mathbf{1}_{E_{2}} T\left(\mathbf{1}_{e_{1}} y\right)=\mathbf{1}_{F\left(e_{1}\right) \cap E_{2}} T(y)$, so that $\left.T(x)\right|_{e_{1} \cap E_{2}}=\left.T(y)\right|_{e_{1} \cap E_{2}} \mu_{2}$-a.e. on $\Omega_{2}$. On the other hand,

$$
\left.T(x)\right|_{\Omega_{2} \backslash E_{2}}=\left.T(y)\right|_{\Omega_{2} \backslash E_{2}}=0,
$$

and hence $T$ is atomic.

\section{Coatomic operators}

Another concept which seems to be interesting to study is given by the following definition. Again, here $X_{i}:=L^{p_{i}}\left(\Omega_{i}, \Sigma_{i}, \mu_{i} ; \mathcal{X}_{i}\right), i=1,2$, and $0 \leqslant p_{i} \leqslant+\infty$.

Definition 4.1. An operator $T: X_{1} \rightarrow X_{2}$ is called coatomic if there is a $\sigma$ homomorphism $\Phi: \Sigma_{2} \rightarrow \Sigma_{1}$ satisfying

$$
\left[\Phi\left(e_{2}\right)\right] \in \underset{T}{\operatorname{Mem}}\left(\tilde{e}_{2}\right)
$$

for all $e_{2} \in \Sigma_{2}$, that is, if $\left.x\right|_{\Phi\left(e_{2}\right)}=\left.y\right|_{\Phi\left(e_{2}\right)}$ implies $\left.T(x)\right|_{e_{2}}=\left.T(y)\right|_{e_{2}}$ for all $x, y \in X_{1}$.

It is not difficult to observe that a notion of a coatomic operator is, in a certain sense, dual to the notion of an atomic operator. However, both classes contain local operators. Certainly, the class of coatomic operators is wider than that of local operators.

Example 4.2. Assume that $g: \Omega_{2} \rightarrow \Omega_{1}$ is a bijection satisfying (2.2) and the inverse function $g^{-1}: \Omega_{1} \rightarrow \Omega_{2}$ has the same property

$$
\mu_{1}\left(g\left(e_{2}\right)\right)=0 \quad \text { for } e_{2} \in \Sigma_{2}, \quad \mu_{2}\left(e_{2}\right)=0 .
$$

Then the respective shift operator $T_{g}: L^{0}\left(\Omega_{1}\right) \rightarrow L^{0}\left(\Omega_{2}\right)$ is coatomic, for one can take $\Phi(e):=g(e)$.

In relation to the latter example we observe that, unlike the class of atomic operators, the class of coatomic operators contains only rather particular shifts, namely, only those described in Example 4.2.

Proposition 4.3. Assume $\left(\Omega_{2}, \Sigma_{2}, \mu_{2}\right)$ is a standard measure space and a $\left(\Sigma_{1}, \Sigma_{2}\right)$ measurable function $g: \Omega_{2} \rightarrow \Omega_{1}$ satisfies (2.2). Then the shift operator $T_{g}: L^{0}\left(\Omega_{1}\right) \rightarrow$ $L^{0}\left(\Omega_{2}\right)$ is coatomic, if and only if $g$ is $\mu_{2}$-equivalent to a bijection and satisfies (4.1). 
Proof. It is only necessity that needs to be proved. Assume that $T_{g}$ is coatomic with respect to a $\sigma$-homomorphism $\Phi: \Sigma_{2} \rightarrow \Sigma_{1}$. Taking $x=\mathbf{1}_{\Phi(e)}$ for an arbitrary $e \in \Sigma_{2}$ we observe that $T_{g}(x)=\mathbf{1}_{g^{-1}(\Phi(e))}$. The definition of coatomic operators then yields $g^{-1}(\Phi(e)) \subset e$. On the other hand, taking $\Omega_{2} \backslash e$ instead of $e$ implies

$$
\Omega_{2} \backslash g^{-1}(\Phi(e))=g^{-1}\left(\Phi\left(\Omega_{2}\right)\right) \backslash g^{-1}(\Phi(e))=g^{-1}\left(\Phi\left(\Omega_{2} \backslash e\right)\right) \subset \Omega_{2} \backslash e,
$$

so that $g^{-1}(\Phi(e))=e$ (up to a $\mu_{2}$-null set), and property (4.1) holds. Since $\left(\Omega_{2}, \Sigma_{2}, \mu_{2}\right)$ is a standard measure space, then by Theorem 32.3 of [16] there is a function $h: \Omega_{1} \rightarrow \Omega_{2}$ satisfying $\Phi\left(e_{2}\right)=h^{-1}\left(e_{2}\right)$ for all Borel sets $e_{2} \subset \Omega_{2}$. Define $\varphi:=h \circ g: \Omega_{2} \rightarrow \Omega_{2}$. Noting that $\left[\varphi^{-1}\left(e_{2}\right)\right]=\left[e_{2}\right]$ for every Borel set $e_{2} \in \Sigma_{2}$, we see that the shift $T_{\varphi}: L^{0}\left(\Omega_{2}\right) \rightarrow$ $L^{0}\left(\Omega_{2}\right)$ is an identity operator in $L^{0}\left(\Omega_{2}\right)$, since it coincides with the identity on the set of simple (i.e. finite-valued) functions. Therefore, $\varphi=T_{\varphi}(\mathrm{id})=$ id $\mu_{2}$-a.e. on $\Omega_{2}$, which proves the statement.

Thus we see that the class of coatomic operators does not coincide with that of atomic operators. Moreover, it is not contained in the latter one, as we will show using the following statement.

Proposition 4.4. Let $\mathcal{X}_{1}$ and $\mathcal{X}_{2}$ be reflexive Banach spaces. A linear bounded operator $T: L^{p}\left(\Omega_{1} ; \mathcal{X}_{1}\right) \rightarrow L^{q}\left(\Omega_{2} ; \mathcal{X}_{2}\right), 1 \leqslant p, q<+\infty$, is coatomic (respectively, atomic), if and only if its adjoint $T^{\prime}: L^{q^{\prime}}\left(\Omega_{2} ; \mathcal{X}_{2}^{\prime}\right) \rightarrow L^{p^{\prime}}\left(\Omega_{1} ; \mathcal{X}_{1}^{\prime}\right)$ is atomic (respectively, coatomic).

Proof. Let $T$ be atomic, while $F: \Sigma_{1} \rightarrow \Sigma_{2}$ is the respective $\sigma$-homomorphism. Consider the identity

$$
\int_{\Omega_{2}}\left\langle(T x)\left(\omega_{2}\right), y^{\prime}\left(\omega_{2}\right)\right\rangle_{2} \mathrm{~d} \mu_{2}\left(\omega_{2}\right)=\int_{\Omega_{1}}\left\langle x\left(\omega_{1}\right),\left(T^{\prime} y^{\prime}\right)\left(\omega_{1}\right)\right\rangle_{1} \mathrm{~d} \mu_{1}\left(\omega_{1}\right),
$$

where $x \in L^{p}\left(\Omega_{1} ; \mathcal{X}_{1}\right), y^{\prime} \in L^{q^{\prime}}\left(\Omega_{2} ; \mathcal{X}_{2}^{\prime}\right)$ and $\langle\cdot, \cdot\rangle_{i}$ is the natural duality between the spaces $\mathcal{X}_{i}$ and $\mathcal{X}_{i}^{\prime}$. We prove that $T^{\prime}$ is coatomic showing that $\left.y^{\prime}\right|_{F\left(e_{1}\right)}=0$ implies $\left.\left(T^{\prime} y^{\prime}\right)\right|_{e_{1}}=0$. Supposing the contrary, we choose according to Corollary A.2.1 of $[\mathbf{1 7}]$ an $x \in L^{p}$ such that

$$
\left\langle x\left(\omega_{1}\right),\left(T^{\prime} y^{\prime}\right)\left(\omega_{1}\right)\right\rangle_{1} \geqslant \frac{1}{2}\left|\left(T^{\prime} y^{\prime}\right)\left(\omega_{1}\right)\right|_{\mathcal{X}_{1}^{\prime}} \quad \text { if } \omega_{1} \in e_{1} \text { and }\left.x\right|_{\Omega_{1} \backslash e_{1}}=0,
$$

thus having $\left.(T x)\right|_{\Omega_{2} \backslash F\left(e_{1}\right)}=0$, and obtain the contradiction

$$
0=\frac{1}{2} \int_{e_{1}}\left|T^{\prime} y^{\prime}\left(\omega_{1}\right)\right|_{\mathcal{X}_{1}^{\prime}} \mathrm{d} \mu_{1}\left(\omega_{1}\right)>0 .
$$

If $T$ is coatomic, $\Phi: \Sigma_{2} \rightarrow \Sigma_{1}$ being the respective $\sigma$-homomorphism, then we are to prove that $\left.y^{\prime}\right|_{e_{2}}=0$ implies $\left.\left(T^{\prime} y^{\prime}\right)\right|_{\Phi\left(e_{2}\right)}=0$. Supposing the contrary, we choose $x$ such that

$$
\left\langle x\left(\omega_{1}\right),\left(T^{\prime} y^{\prime}\right)\left(\omega_{1}\right)\right\rangle_{1} \geqslant \frac{1}{2}\left|\left(T^{\prime} y^{\prime}\right)\left(\omega_{1}\right)\right|_{\mathcal{X}_{1}^{\prime}} \quad \text { if } \omega_{1} \in \Phi\left(e_{2}\right) \text { and }\left.x\right|_{\Phi\left(\Omega_{2} \backslash e_{2}\right)}=0,
$$


thus having $\left.(T x)\right|_{\Omega_{2} \backslash e_{2}}=0$, and obtain the contradiction

$$
0=\frac{1}{2} \int_{\Phi\left(e_{2}\right)}\left|T^{\prime} y^{\prime}\left(\omega_{1}\right)\right|_{\mathcal{X}_{1}^{\prime}} \mathrm{d} \mu_{1}\left(\omega_{1}\right)>0 .
$$

The 'only if' part of the statement is proved in a completely symmetric way by interchanging $T$ and $T^{\prime}$.

From the latter proposition, which is of independent interest, we may conclude that, roughly speaking, while the class of atomic operators contains all the shift operators between Lebesgue spaces, the class of coatomic operators contains all their adjoints. Now we are able to show that there are coatomic operators which are not atomic.

Example 4.5. Let $\Omega=(0,1), \Sigma$ be the $\sigma$-algebra of Lebesgue-measurable subsets of $(0,1)$, and $\mu$ be the Lebesgue measure. Consider the function

$$
g(\omega):=\sin ^{2}(1 / \omega)
$$

The corresponding shift operator $T_{g}: L^{\infty}(\Omega) \rightarrow L^{\infty}(\Omega)$ is order continuous and, therefore, continuous in *-weak topology $\sigma\left(L^{\infty}, L^{1}\right)$. Then there is a dual operator (i.e. adjoint with respect to the duality) $T: L^{1}(\Omega) \rightarrow L^{1}(\Omega)$ such that $T^{\prime}=T_{g}$. As it has been proven that $T_{g}$ is atomic (being a shift), then, by Proposition 4.4, we have that $T$ is coatomic. However, it cannot be atomic, because otherwise, by the same proposition, $T_{g}$ would be coatomic.

Much like the class of atomic operators, the class of coatomic operators is also obviously closed with respect to compositions, but not closed with respect to finite sums. In fact, the operator $T: L^{2}(\Omega) \rightarrow L^{2}(\Omega)$ defined in Example 3.4 is self-adjoint. Since it is not atomic, it is by Proposition 4.4 not coatomic either, although both of the summands are.

We now give an interesting example of a coatomic operator arising in probability theory.

Example 4.6. Let $\Sigma_{1} \subset \Sigma_{2}$ be two different $\sigma$-algebras on the same set $\Omega$, the probability $\mathbb{P}$ being defined on $\Sigma_{2}$. For every $1 \leqslant p \leqslant \infty$, the natural imbedding $\imath$ : $L^{p}\left(\Omega, \Sigma_{1}, \mathbb{P}\right) \rightarrow L^{p}\left(\Omega, \Sigma_{2}, \mathbb{P}\right)$ is an atomic operator, the respective $\sigma$-homomorphism $F: \Sigma_{1} \rightarrow \Sigma_{2}$ being induced by the imbedding of $\Sigma_{1}$ into $\Sigma_{2}$. Its adjoint, which by Proposition 4.4 is coatomic, is the operator of conditional expectation $\mathcal{E}\left(\cdot \mid \Sigma_{1}\right)$.

\subsection{Representation}

The following assertion gives the relation between coatomic operators, local operators and shifts.

Theorem 4.7. For every coatomic operator $T: L^{0}\left(\Omega_{1} ; \mathcal{X}_{1}\right) \rightarrow L^{0}\left(\Omega_{2} ; \mathcal{X}_{2}\right)$, the operator

$$
N:=T \circ T_{\Phi}: L^{0}\left(\Omega_{2} ; \mathcal{X}_{1}\right) \rightarrow L^{0}\left(\Omega_{2} ; \mathcal{X}_{2}\right),
$$

where $\Phi: \Sigma_{2} \rightarrow \Sigma_{1}$ is the respective $\sigma$-homomorphism, is local. The continuity of $T$ (in measure), implies that of the operator $N$. Moreover, if $\left(\Omega_{1}, \Sigma_{1}, \mu_{1}\right)$ is a standard measure 
space, then there is a $\left(\Sigma_{2}, \Sigma_{1}\right)$-measurable function $g: \Omega_{1} \rightarrow \Omega_{2}$ satisfying (2.2), such that $N=T \circ T_{g}$.

Proof. The generalized shift $T_{\Phi}: L^{0}\left(\Omega_{2} ; \mathcal{X}_{1}\right) \rightarrow L^{0}\left(\Omega_{1} ; \mathcal{X}_{1}\right)$ is defined and is automatically continuous by Lemma 3.7 . It is clear now that $N=T \circ T_{\Phi}: L^{0}\left(\Omega_{1} ; \mathcal{X}_{1}\right) \rightarrow L^{0}\left(\Omega_{2} ; \mathcal{X}_{2}\right)$ is local. The continuity of $N$ is evidently implied by that of $T$. If $\left(\Omega_{1}, \Sigma_{1}, \mu_{1}\right)$ is a standard measure space, then, as in the proof of Theorem 3.8, we construct a $\left(\Sigma_{2}, \Sigma_{1}\right)$-measurable function $g: \Omega_{1} \rightarrow \Omega_{2}$ satisfying

$$
\tilde{\Phi}\left(\tilde{e}_{2}\right)=\left[g^{-1}\left(e_{2}\right)\right] \text { for all } e_{2} \in \Sigma_{2},
$$

and hence also (2.2), and observe that the latter relationship implies $T_{\Phi}=T_{g}$.

Corollary 4.8. Let $\left(\Omega_{1}, \Sigma_{1}, \mu_{1}\right)$ be a standard measure space, $\mathcal{X}_{i}$ be separable metric spaces. Under the continuum hypothesis one can find for every atomic operator $T$ : $L^{0}\left(\Omega_{1} ; \mathcal{X}_{1}\right) \rightarrow L^{0}\left(\Omega_{2} ; \mathcal{X}_{2}\right)$ a measurable function $g: \Omega_{2} \rightarrow \Omega_{1}$ satisfying (2.2) and a function $f: \Omega_{1} \times \mathcal{X}_{1} \rightarrow \mathcal{X}_{2}$ such that $T \circ T_{g}=N_{f}$, i.e. for every $x \in L^{0}\left(\Omega_{2} ; \mathcal{X}_{1}\right)$, one has

$$
(T x)\left(g\left(\omega_{2}\right)\right)=f\left(\omega_{2}, x\left(\omega_{2}\right)\right) \quad \mu_{2} \text {-a.e. on } \Omega_{2} .
$$

Moreover, if $T$ is continuous in measure, then (independently of the validity of continuum hypothesis) the function $f$ may be assumed a Carathéodory function.

\subsection{Acting, boundedness and continuity}

Unlike atomic operators, but similar to Nemytskiĩ operators, the coatomic operators possess better continuity properties in Lebesgue spaces. In this subsection we assume that $\mu_{1}$ and $\mu_{2}$ are non-atomic and that $\mathcal{X}_{1}$ and $\mathcal{X}_{2}$ are separable Banach spaces.

Theorem 4.9. If a coatomic operator $T: L^{p}\left(\Omega_{1} ; \mathcal{X}_{1}\right) \rightarrow L^{q}\left(\Omega_{2} ; \mathcal{X}_{2}\right)$, where $1 \leqslant p$, $q<\infty$, maps $L^{p}$-convergent sequences into measure convergent ones, then it is continuous as an operator between these two spaces.

\section{Proof.}

Step 1. Assume without loss of generality that $T(0)=0$, and suppose that continuity, asserted in the theorem, fails. Then there is a converging sequence $\left\{x_{\nu}\right\} \subset L^{p}\left(\Omega_{1} ; \mathcal{X}_{1}\right)$ such that the sequence $\left\{T\left(x_{\nu}\right)\right\}$ does not converge in $L^{q}\left(\Omega_{2} ; \mathcal{X}_{2}\right)$. Denote

$$
\gamma(\delta):=\sup \left\{\left\|\mathbf{1}_{e_{1}} x_{\nu}\right\|_{p}, e_{1} \in \Sigma_{1}, \mu_{1}\left(e_{1}\right)<\delta, \nu \in \mathbb{N}\right\},
$$

where $\|\cdot\|_{p}$ stands for the norm in the space $L^{p}\left(\Omega_{1} ; \mathcal{X}_{1}\right)$. Since $\left\{x_{\nu}\right\}$ is norm convergent, hence equi-integrable, we have that $\gamma(\delta) \rightarrow 0$ as $\delta \rightarrow 0$. Moreover, since $\left\{T\left(x_{\nu}\right)\right\} \subset$ $L^{q}\left(\Omega_{2} ; \mathcal{X}_{2}\right)$ is not convergent, we can choose an appropriate subsequence of $\left\{x_{\nu}\right\}$, denoted further by the same index, and a respective family $\left\{E_{\nu}\right\} \subset \Sigma_{2}$, so that for some $\varepsilon>0$ one has

$$
\left\|\mathbf{1}_{E_{\nu}} T\left(x_{\nu}\right)\right\|_{q}>\varepsilon, \quad \text { while } \gamma\left(\mu_{1}\left(\Phi\left(E_{\nu}\right)\right)\right)<2^{-\nu} \text { and } \mu_{2}\left(E_{\nu}\right)<2^{-\nu} .
$$


Step 2. Define by induction a sequence $\left\{G_{k}\right\} \subset \Sigma_{2}$ such that

(i) $G_{k}$ are pairwise disjoint;

(ii) $\mu_{2}\left(G_{k}\right) \rightarrow 0$ as $k \rightarrow \infty$;

(iii) $\left\|\mathbf{1}_{G_{k}} T\left(x_{\nu_{k}}\right)\right\|_{q}>\varepsilon / 2$ for some subsequence $\left\{x_{\nu_{k}}\right\}$; and

(iv) $\left(\bigcup_{j=1}^{k} G_{j}\right) \bigcap\left(\bigcup_{j=\nu_{k}}^{\infty} E_{j}\right)=\emptyset$ for some $\nu_{k} \in \mathbb{N}$.

If $k=1$, choose $G_{1}:=E_{1} \backslash \bigcup_{i=\nu_{1}}^{\infty} E_{\nu}$, where $\nu_{1} \in \mathbb{N}$ is large enough to provide

$$
\left\|\mathbf{1}_{\cup_{j=\nu_{1}}^{\infty} E_{j}} T\left(x_{1}\right)\right\|_{q}<\varepsilon / 2 .
$$

The choice of such $\nu_{1} \in \mathbb{N}$ is possible, since by construction $\mu_{2}\left(\bigcup_{j=\nu}^{\infty} E_{j}\right) \rightarrow 0$. If there already exists a family $G_{1}, \ldots, G_{k}$ satisfying (i)-(iv), then we can again find a number $\nu_{k+1}$ such that

$$
\left\|\mathbf{1}_{\cup_{j=\nu_{k+1}}^{\infty} E_{j}} T\left(x_{k+1}\right)\right\|_{q}<\varepsilon / 2
$$

and then we set

$$
G_{k+1}:=E_{\nu_{k}} \backslash \bigcup_{\nu=\nu_{k+1}}^{\infty} E_{\nu}
$$

Step 3. Since $\gamma\left(\mu_{1}\left(\Phi\left(E_{\nu}\right)\right)\right)<2^{-\nu}$, we also have that $\sum_{k=1}^{\infty} \gamma\left(\mu_{1} \Phi\left(G_{k}\right)\right)<\infty$. Therefore, a measurable function $y:=\sum_{k=1}^{\infty} \mathbf{1}_{\Phi\left(G_{k}\right)} x_{\nu_{k}}$ belongs to the space $L^{p}\left(\Omega_{1} ; \mathcal{X}_{1}\right)$, because

$$
\|y\|_{p} \leqslant \sum_{k=1}^{\infty}\left\|\mathbf{1}_{\Phi\left(G_{k}\right)} x_{\nu_{k}}\right\|_{p} \leqslant \sum_{k=1}^{\infty} \gamma\left(\mu_{1}\left(\Phi\left(G_{k}\right)\right)\right)<\infty .
$$

On the other hand, since $T$ is coatomic, one has

$$
\|T(y)\|_{q}^{q}=\left\|\sum_{k=1}^{\infty} \mathbf{1}_{G_{k}} T\left(x_{\nu_{k}}\right)\right\|_{q}^{q}=\sum_{k=1}^{\infty}\left\|\mathbf{1}_{G_{k}} T\left(x_{\nu_{k}}\right)\right\|_{q}^{q}=\infty,
$$

because each summand in the right-hand side is greater than $\varepsilon / 2$. This contradiction completes the proof.

\subsection{Non-compactness}

The coatomic operators acting in ideal function spaces possess, similarly to local and atomic operators, the property of non-compactness.

Theorem 4.10. Assume $0 \leqslant p, q \leqslant \infty$ and that the $\mathcal{X}_{i}$ are separable Banach spaces. A non-constant coatomic operator $T: L^{p}\left(\Omega_{1} ; \mathcal{X}_{1}\right) \rightarrow L^{q}\left(\Omega_{2} ; \mathcal{X}_{2}\right), 1 \leqslant p, q \leqslant+\infty$, is not compact if $\mu_{2}$ is a non-atomic measure. 
Proof. Again, as in the proof of Theorem 3.13, we choose $x \in L^{p}\left(\Omega_{1} ; \mathcal{X}_{1}\right)$ and $E_{2} \in$ $\Sigma_{2}, \mu_{2}\left(E_{2}\right)>0$, such that $(T(x))\left(\omega_{2}\right) \neq(T(0))\left(\omega_{2}\right)$ for all $\omega_{2} \in E_{2}$, and assume without loss of generality that $T(0)=0$. Now in a completely analogous way with the proof of Theorem 3.13 construct a sequence $\left\{E_{2}(k)\right\}_{k=1}^{\infty}$ of measurable subsets of $E_{2}$ with the properties

$$
\mu_{2}\left(E_{2}(k)\right)=\mu_{2}\left(E_{2}\right) / 2 \quad \text { and } \quad \mu_{2}\left(E_{2}(k) \Delta E_{2}(j)\right)=\mu_{2}\left(E_{2}\right) / 2
$$

for all $k \in \mathbb{N}$ and for all $j \in \mathbb{N}, j \neq k$. The sequence of functions $x_{k}:=\mathbf{1}_{\Phi\left(E_{2}(k)\right)} x$ is bounded in $L^{p}\left(\Omega_{1} ; \mathcal{X}_{1}\right)$, but the sequence of the images $T\left(x_{k}\right)$ is not precompact in $L^{q}\left(\Omega_{2} ; \mathcal{X}_{2}\right)$, because

$$
\left|\left(T x_{j}\right)\left(\omega_{2}\right)-\left(T x_{k}\right)\left(\omega_{2}\right)\right|_{\mathcal{X}_{2}}=\mathbf{1}_{E_{2}(j) \Delta E_{2}(k)}|T(x)|_{\mathcal{X}_{2}},
$$

while for $j<k$ the latter is equal to $|T(x)|_{\mathcal{X}_{2}}>0$ on the set $E_{2}(j) \Delta E_{2}(k)$ of the measure $\mu_{2}\left(E_{2}\right) / 2$.

\subsection{Weak continuity}

Like both local and atomic operators, coatomic operators are, as a rule, degenerate in weak topology of $L^{p}$.

Theorem 4.11. Let $1 \leqslant p, q<\infty, \mu_{2}$ be a non-atomic measure and $\mathcal{X}_{i}$ be separable Banach spaces. Then a coatomic operator $T: L^{p}\left(\Omega_{1} ; \mathcal{X}_{1}\right) \rightarrow L^{q}\left(\Omega_{2} ; \mathcal{X}_{2}\right)$ is weakly continuous, if and only if $T$ is affine, i.e. $T(\cdot)-T(0)$ is a linear bounded operator.

The proof is completely analogous to that of Theorem 3.14.

\subsection{Convergence}

Convergence properties of coatomic operators are much worse than those of atomic operators. First we consider linear coatomic operators.

Example 4.12. Let $\Omega=(0,1)$ and the functions $g^{\nu}: \Omega \rightarrow \mathbb{R}, \nu \in \mathbb{N}$, be defined as

$$
g^{\nu}(\omega)=\nu \omega-j, \quad j / \nu \leqslant \omega<(j+1) / \nu, \quad j=0, \ldots, \nu-1 .
$$

In [9] it has been noted that the operators $T_{g^{\nu}}: L^{2} \rightarrow L^{2}$ converge to an operator $T: L^{2} \rightarrow L^{2}$ given by the formula

$$
(T x)(\omega):=\int_{\Omega} x\left(\omega^{\prime}\right) \mathrm{d} \omega^{\prime} \cdot 1(\omega)
$$

in such a way that their duals $T_{g^{\nu}}^{\prime}: L^{2} \rightarrow L^{2}$ converge strongly (pointwise) to the dual $T^{\prime}: L^{2} \rightarrow L^{2}$ of the operator $T$. In fact, to prove this convergence, it is enough to verify that for every weakly convergent sequence $\left\{x_{\nu}\right\} \subset L^{2}, x_{\nu} \rightarrow x$, one has $T_{g^{\nu}} x_{\nu} \rightarrow T x$ (see $[\mathbf{9}]$ ). We see now that all $T_{g^{\nu}}^{\prime}$ are coatomic as duals to the shift operators, while their strong limit $T^{\prime}=T$ is obviously not coatomic. 
Nevertheless, the following proposition concerning convergence of a sequence of linear coatomic operators can be obtained.

Proposition 4.13. A uniform limit $T$ of a sequence of linear bounded coatomic operators $T_{\nu}: L^{p}\left(\Omega_{1} ; \mathcal{X}_{1}\right) \rightarrow L^{q}\left(\Omega_{2} ; \mathcal{X}_{2}\right)$, where $1 \leqslant p, q<\infty$, is coatomic.

Proof. The assumptions imply that the sequence of duals $\left\{T_{\nu}^{\prime}\right\}$ converges strongly to a bounded linear operator $T^{\prime}$. By the Proposition 4.4, the operators $T_{\nu}^{\prime}$ are atomic and, therefore, by Theorem 3.15, so is $T^{\prime}$. Again applying Proposition 4.4, we complete the proof.

Unfortunately, nothing can be said about convergence of nonlinear coatomic operators, as the example below shows.

Example 4.14. Take $\Omega_{1}:=\mathbb{N}, \Omega_{2}:=\{0\} \cup \mathbb{N}, \Sigma_{i}:=2^{\Omega_{i}}, \mu_{1}(\{i\}):=2^{-i}\left(i \in \Omega_{1}\right)$, $\mu_{2}(\{i\}):=2^{-i}\left(i \in \Omega_{2}\right)$. Consider some continuous function $\varphi: \mathbb{R} \rightarrow[0,1]$ and define the sequence of operators $T_{\nu}: L^{p}\left(\Omega_{1} ; \mathbb{R}\right) \rightarrow L^{q}\left(\Omega_{2} ; \mathbb{R}\right)$ by the relationships

$$
\bar{T}_{\nu}\left(\left\{\xi_{i}\right\}\right):=2^{-\nu-1}\left\{2^{\nu+1} \bar{\xi}_{\nu}, \xi_{\nu+1}, \xi_{\nu+2}, \ldots\right\} \quad \text { and } \quad T_{\nu}\left(\left\{\xi_{i}\right\}\right):=\bar{T}_{\nu}\left(\left\{\varphi\left(\xi_{i}\right)\right\}\right) .
$$

Here $\left\{\xi_{i}\right\}$ is an arbitrary element from $L^{p}\left(\Omega_{1}, \Sigma_{1}, \mu_{1} ; \mathcal{X}_{1}\right)$ which consists of sequences indexed by the natural numbers, while $T_{\nu}\left(\left\{\xi_{i}\right\}\right)$ is a sequence indexed by the non-negative integers,

$$
\bar{\xi}_{\nu}:=\frac{\sum_{i=1}^{\nu} 2^{-i} \xi_{i}}{1-2^{-\nu-1}}
$$

Clearly, the sequence of the operators $T_{\nu}$ converges uniformly to the operator

$$
T\left(\left\{\xi_{i}\right\}\right):=\{\bar{\xi}, 0,0, \ldots\},
$$

where $\bar{\xi}=\sum_{i=1}^{\infty} 2^{-i} \varphi\left(\xi_{i}\right)$, which is obviously not coatomic. On the other hand, $T_{\nu}$ is coatomic with respect to the $\sigma$-homomorphism defined by

$$
\Phi_{\nu}(\{i\}):= \begin{cases}\{i+\nu\}, & i \geqslant 1, \\ \{1,2, \ldots, \nu\}, & i=0 .\end{cases}
$$

Acknowledgements. M.E.D.'s research has been partly supported by the Israeli Ministry of Science and the Center for Scientific Absorption of the Ministry of Absorption.

\section{References}

1. J. ApPell AND P. P. ZABREJKo, Nonlinear superposition operators, Cambridge Tracts in Mathematics, vol. 95 (Cambridge University Press, 1990).

2. L. Arnold And V. Wintutz, Stationary solutions of linear systems with additive and multiplicative noise, Stochastics 7 (1982), 133-155.

3. N. V. Azbelev, V. P. Maksimov, and L. F. Rakhmatullina, Introduction to the theory of functional differential equations (Nauka, Moscow, 1991). (In Russian.)

4. D. L. Cohn, Measure theory (Birkhäuser, Boston, 1980).

5. J. L. Doob, Stochastic processes (Wiley, 1953). 
6. J. L. Doob, Measure theory, Graduate Texts in Mathematics, vol. 143 (Springer, 1993).

7. M. E. DrakHLin, On nonlinear inner superposition operator, in Boundary value problems, pp. 76-81 (Perm Polytechnical Institute, Perm, 1986). (In Russian.)

8. M. E. Drakhlin and E. Stepanov, Convergence of composition operators and optimal control problems, J. Nonlin. Analysis 30 (1997), 505-512.

9. M. E. Drakhlin AND E. Stepanov, Weak convergence of inner superposition operators, Proc. Am. Math. Soc. 126 (1997), 173-179.

10. P. R. Halmos And J. von Neumann, Operator methods in classical mechanics, II, Ann. Math. 43 (1942), 332-350.

11. M. A. Krasnosel'SkII AND A. V. Pokrovskǐ̌, On discontinuous superposition operator. Usp. Mat. Nauk 32 (1977), 169-170. (In Russian.)

12. S. E. A. Mohammed, Stochastic functional differential equations, Research Notes in Mathematics, vol. 99 (Pitman, Boston, MA, 1984).

13. A. Ponosov, On the Nemytskiǐ conjecture. Sov. Acad. Sci. Dokl. Math. 289 (1986), 1308-1311. (In Russian. English translation: Sov. Math. Dokl. 34 (1987), 231-233.)

14. A. Ponosov, A fixed point method in the theory of stochastic differential equations. Sov. Acad. Sci. Dokl. Math. 299 (1988), 562-585. (In Russian. English translation: Sov. Math. Dokl. 37 (1988), 426-429.)

15. I. V. Shragin, Abstract Nemyckiǐ operators are locally defined operators, Sov. Acad. Sci. Dokl. Math. 227 (1976), 47-49. (In Russian. English translation: Sov. Math. Dokl. 17 (1976), 354-357.)

16. R. SiKORSKI, Boolean algebras (Springer, 1960).

17. M. VÄтн, Ideal spaces, Lecture Notes in Mathematics, vol. 1664 (Springer, 1997).

18. J. von Neumann, Einige Sätze über die meßbaren Abbildungen, Ann. Math. 33 (1932), 574-586. (In German.)

19. I. VRKoč, The representation of Carathéodory operators, Czech. Math. J. 19 (1969), 99-109.

20. L. WEIS, On representation of order continuous operators by random measures. Trans. Am. Math. Soc. 285 (1984), 535-563. 